Rev. Mat. Iberoam. 28 (2012), no. 1, 239-272

(C) European Mathematical Society

DOI $10.4171 / \mathrm{RMI} / 676$

\title{
Composition operators on Besov algebras
}

\author{
Madani Moussai
}

Abstract. We study the composition operator $T_{f}(g):=f \circ g$ on Besov spaces $B_{p, q}^{s}(\mathbb{R})$. In the case $1<p<+\infty, p \leq q \leq+\infty$ and $s>1+(1 / p)$ we will prove that the operator $T_{f}$ takes $B_{p, q}^{s}(\mathbb{R})$ into itself if and only if $f(0)=0$ and $f$ belongs locally to $B_{p, q}^{s}(\mathbb{R})$.

\section{Introduction}

For a Borel function $f: \mathbb{R} \rightarrow \mathbb{R}$, we are interested in the associated nonlinear composition operator $T_{f}: g \rightarrow f \circ g$ on the Besov space $B_{p, q}^{s}(\mathbb{R})$ into itself. The Superposition Operator Problem (S.O.P.) for a given real valued function space $E$ consists in the full characterization of functions $f$ such that $T_{f}(E) \subset E$. The following results are well known:

- If $1<p<+\infty, s>n / p$ and $f \in C^{\infty}(\mathbb{R})$, then $T_{f}$ takes the Bessel potential spaces $H_{p}^{s}\left(\mathbb{R}^{n}\right)$ (resp. $\left.B_{p, q}^{s}\left(\mathbb{R}^{n}\right)\right)$ into itself, see e.g. Meyer [12], (resp. Peetre [13]).

- Dahlberg [9] proved that for $1 \leq p \leq+\infty$ and $1+(1 / p)<m<n / p(m$ integer), if $T_{f}$ maps the Sobolev spaces $W_{p}^{m}\left(\mathbb{R}^{n}\right)$ into itself, then $f$ is a linear function. Also, after the work of Marcus and Mizel [11] on $W_{p}^{1}\left(\mathbb{R}^{n}\right)$, Bourdaud [2] proved that for either $m \geq 2$ and $m p>n$, or $m=n \geq 2$ and $p=1$, the operator $T_{f}$ maps $W_{p}^{m}\left(\mathbb{R}^{n}\right)$ into itself if and only if $f \in W_{p}^{m, \ell o c}(\mathbb{R})$.

- The result of Dahlberg has been extended to $B_{p, q}^{s}\left(\mathbb{R}^{n}\right)$. More precisely, for $1 \leq p, q \leq+\infty$ and either $1+(1 / p)<s<n / p$, or $1+(1 / p)=s<n / p$ and $1<q \leq+\infty$, if the operator $T_{f}$ acts on $B_{p, q}^{s}\left(\mathbb{R}^{n}\right)$ then $f(t)=c t$ for some real $c$, see e.g. [15] and [16].

The characterization of those functions $f$ such that $T_{f}$ acts on $B_{p, q}^{s}\left(\mathbb{R}^{n}\right)$ requires to investigate the necessary conditions. In this sense, we recall the following two results:

Mathematics Subject Classification (2010): 46E35, 47H30.

Keywords: Littlewood-Paley decomposition, Besov spaces, composition operator, functions of bounded $p$-variation. 
(r1) Let $s>0$ and $1 \leq p, q \leq+\infty$. If $T_{f}$ takes $B_{p, q}^{s}\left(\mathbb{R}^{n}\right) \cap L_{\infty}\left(\mathbb{R}^{n}\right)$ to $B_{p, \infty}^{s}\left(\mathbb{R}^{n}\right)$, then $f$ is locally Lipschitz continuous, i.e., $f \in W_{\infty}^{1, \ell \text {, }}(\mathbb{R})$.

(r2) Let $s>0$ and $1 \leq p, q \leq+\infty$. If $T_{f}$ takes the Schwartz space $\mathcal{S}\left(\mathbb{R}^{n}\right)$ to $B_{p, q}^{s}\left(\mathbb{R}^{n}\right)$, then $f \in B_{p, q}^{s, \ell o c}(\mathbb{R})$.

In (r1) we have chosen $B_{p, \infty}^{s}\left(\mathbb{R}^{n}\right)$ since the embedding $B_{p, q}^{s}\left(\mathbb{R}^{n}\right) \hookrightarrow B_{p, \infty}^{s}\left(\mathbb{R}^{n}\right)$ is satisfied for all $q \geq 1$. Result (r1) is proved in [16] or [1] for instance; the proof of $(\mathrm{r} 2)$ can be found in 5.3.1-Theorem 2 of [16]. Now (r1) and (r2) lead to the following conjecture:

Conjecture 1.1. Let $f: \mathbb{R} \rightarrow \mathbb{R}$. The composition operator $T_{f}$ takes the space $B_{p, q}^{s}\left(\mathbb{R}^{n}\right) \cap L_{\infty}\left(\mathbb{R}^{n}\right)$ into $B_{p, q}^{s}\left(\mathbb{R}^{n}\right)$ if only if the following three conditions hold:
(i) $f(0)=0$
(ii) $f \in W_{\infty}^{1, \ell o c}(\mathbb{R})$,
(iii) $f \in B_{p, q}^{s, \ell o c}(\mathbb{R})$.

The necessity of (i) is obvious in case $p<+\infty$ and can be obtained by testing $f$ on the zero function. However (ii) and (iii) can be simplified in the following subcases:

- If $s>1+(1 / p)$, then $B_{p, q}^{s}(\mathbb{R}) \hookrightarrow W_{\infty}^{1}(\mathbb{R})$, hence the condition (ii) can be dropped.

- If $0<s<1$, then $W_{\infty}^{1, \ell o c}(\mathbb{R}) \hookrightarrow W_{p}^{1, \ell o c}(\mathbb{R}) \hookrightarrow B_{p, q}^{s, \ell o c}(\mathbb{R})$, hence the condition (iii) can be dropped. The above conjecture has been proved in this case, see Bourdaud [3].

In this paper we are interested in the case of Besov spaces on $\mathbb{R}$, when the parameters $s$ and $p$ satisfy the condition $s>1+(1 / p)$. We will establish superposition theorems in which the functions $f$, associated to the composition operator, belong locally to Besov space. The proof of this result relies on sharp estimates using a weaker condition for $f$, that is $f^{\prime} \in L_{\infty}(\mathbb{R}) \cap \dot{B}_{p, q}^{s-1}(\mathbb{R})$, where the dotted space represents the homogeneous space. Notice that we will use, in particular, embeddings in Besov space, and some properties of Wiener space $B V_{p}(\mathbb{R})$ of functions of bounded $p$-variation [20]. The method presented here has been used in case of Lizorkin-Triebel spaces [8].

For more information about S.O.P., we refer the reader to the different works of Bourdaud and his collaborators ([2], [3], [4] and [5] for example), and the papers [6] and [7] in which we have partially solved the S.O.P. in both Besov and LizorkinTriebel spaces.

\section{Notation and plan of the paper}

We work with functions defined on the Euclidean space $\mathbb{R}^{n}$. However, in Sections $4-6$ we will omit $\mathbb{R}$ in the notation, since all the functions are defined on the real line $\mathbb{R}$, and assumed to be real valued. For $s \in \mathbb{R},[s]$ denotes the greatest integer less than or equal to $s$. With $\|\cdot\|_{p}$ we denote the $L_{p}$-norm on $\mathbb{R}^{n}$. We denote by $C_{b}\left(\mathbb{R}^{n}\right)$ the Banach space of bounded continuous functions on $\mathbb{R}^{n}$, endowed with the supremum, and by $C_{u b}\left(\mathbb{R}^{n}\right)$ the Banach space of bounded and uniformly 
continuous functions on $\mathbb{R}^{n}$. We denote by $C_{0}\left(\mathbb{R}^{n}\right)$ the Banach subspace of $C_{b}\left(\mathbb{R}^{n}\right)$ with 0 limiting value at infinity. Let $\mathcal{D}\left(\mathbb{R}^{n}\right)$ (respectively, $\mathcal{S}\left(\mathbb{R}^{n}\right)$ and $\mathcal{S}^{\prime}\left(\mathbb{R}^{n}\right)$ ) denote the $C^{\infty}$-functions with compact support (respectively, the Schwartz space of all rapidly decreasing $C^{\infty}$-functions and its topological dual) on $\mathbb{R}^{n}$. We define the difference operators by $\Delta_{h} f:=\Delta_{h}^{1} f=f(\cdot+h)-f$ and $\Delta_{h}^{m} f:=\Delta_{h}\left(\Delta_{h}^{m-1} f\right)$, $m=2,3, \ldots, h \in \mathbb{R}^{n}$.

We denote by $\rho$ a (cut-off) function in $\mathcal{D}\left(\mathbb{R}^{n}\right)$, such that

$$
0 \leq \rho \leq 1, \quad \rho(x)=1 \text { for }|x| \leq 1, \quad \rho(x)=0 \text { for }|x| \geq 3 / 2 .
$$

We put $\rho_{t}(x):=\rho(x / t), t>0$. The function $\rho$ depends on $n$.

If $E$ is a Banach function space on $\mathbb{R}^{n}$ we denote by $E^{\ell o c}$ the collection of all functions $f$ such that $\varphi f \in E$ for all $\varphi \in \mathcal{D}\left(\mathbb{R}^{n}\right)$.

A mapping $T: E \rightarrow E$ is called sublinear if there exists a constant $c>0$ such that

$$
\|T f\|_{E} \leq c\left(1+\|f\|_{E}\right), \quad \forall f \in E .
$$

Throughout the paper, constants $c, c_{1}, \ldots$ are strictly positive and depend only on the fixed parameters $n, s, p$ and $q$; their values may vary from line to line.

The paper is organized as follows. In Section 2 we formulate the main results, and add a short comment. In Section 3, we first collect in Subsections 3.2-3.3 the needed properties of the homogeneous and inhomogeneous Besov spaces. Secondly, as $L_{\infty}\left(\mathbb{R}^{n}\right) \cap \dot{B}_{p, q}^{s}\left(\mathbb{R}^{n}\right)$ plays an important role in this work, we give, in Subsection 3.4, an explicit characterization of this space. Section 4 is devoted to the proof of the sharp estimate of composition operators, which will be given according to the cases $1+(1 / p)<s<2$, and $s=2$, and $2<s \leq 2+(1 / p)$, and is the essential part of the paper. In Section 5 we give the proof of our main results. Finally, the optimality of the exponent $s-(1 / p)$, which appears in the right hand side of the sharp estimate, will be studied in Section 6.

\section{Statement of the main results}

Our main results on the S.O.P. consist of the following two theorems:

Theorem 2.1. Let $1<p<+\infty, p \leq q \leq+\infty$ and $s>1+(1 / p)$.

(i) For a Borel measurable function $f: \mathbb{R} \rightarrow \mathbb{R}$ the composition operator $T_{f}$ acts on $B_{p, q}^{s}(\mathbb{R})$ if and only if $f(0)=0$ and $f \in B_{p, q}^{s, l o c}(\mathbb{R})$.

(ii) If $f(0)=0$ and $f \in B_{p, q}^{s, \ell o c}(\mathbb{R})$, then the operator $T_{f}: B_{p, q}^{s}(\mathbb{R}) \rightarrow B_{p, q}^{s}(\mathbb{R})$ is bounded but not sublinear, unless $f$ itself is linear.

We introduce the space of all functions in $L_{\infty}(\mathbb{R}) \cap \dot{B}_{p, q}^{s}(\mathbb{R})$, which is denoted by $\mathcal{B}_{p, q}^{s}(\mathbb{R})$ and defined by

$$
\|f\|_{\mathcal{B}_{p, q}^{s}(\mathbb{R})}:=\|f\|_{\infty}+\|f\|_{\dot{B}_{p, q}^{s}(\mathbb{R})}<+\infty .
$$


Theorem 2.2. Let $s, p, q$ be real numbers as in Theorem 2.1. Then there exists a constant $c>0$ such that the inequality

$$
\left\|(f \circ g)^{\prime}\right\|_{\mathcal{B}_{p, q}^{s-1}(\mathbb{R})} \leq c\left\|f^{\prime}\right\|_{\mathcal{B}_{p, q}^{s-1}(\mathbb{R})}\left(\|g\|_{B_{p, q}^{s}(\mathbb{R})}+\|g\|_{B_{p, q}^{s}(\mathbb{R})}^{s-(1 / p)}\right)
$$

holds for all functions $f$ such that $f^{\prime} \in \mathcal{B}_{p, q}^{s-1}(\mathbb{R})$, and all $g \in B_{p, q}^{s}(\mathbb{R})$.

As an immediate consequence we have the following corollary:

Corollary 2.3. Let $s, p, q$ be real numbers as in Theorem 2.1.

(i) There exists a constant $c>0$ such that the inequality

$$
\|f \circ g\|_{B_{p, q}^{s}(\mathbb{R})} \leq c\left\|f^{\prime}\right\|_{\mathcal{B}_{p, q}^{s-1}(\mathbb{R})}\left(\|g\|_{B_{p, q}^{s}(\mathbb{R})}+\|g\|_{B_{p, q}^{s}(\mathbb{R})}^{s-(1 / p)}\right)
$$

holds for all functions $f: \mathbb{R} \rightarrow \mathbb{R}$ such that $f^{\prime} \in \mathcal{B}_{p, q}^{s-1}(\mathbb{R})$ and $f(0)=0$, and all $g \in B_{p, q}^{s}(\mathbb{R})$.

(ii) There exists a constant $c>0$ such that the inequality (2.2) holds for all functions $f \in B_{p, q}^{s, \ell o c}(\mathbb{R})$ satisfying $f(0)=0$ and all $g \in B_{p, q}^{s}(\mathbb{R})$, with $\left\|\left(f \rho_{\|g\|_{\infty}}\right)^{\prime}\right\|_{\mathcal{B}_{p, q}^{s-1}(\mathbb{R})}$ instead of $\left\|f^{\prime}\right\|_{\mathcal{B}_{p, q}^{s-1}(\mathbb{R})}$.

Remark 2.4. Let $s, p$, and $q$ be as in Theorem 2.1. Let $\mathcal{M}$ be a bounded set in $B_{p, q}^{s}(\mathbb{R})$. Then from the continuous embedding $B_{p, q}^{s}(\mathbb{R}) \hookrightarrow C_{b}(\mathbb{R})$ we conclude that $\mathcal{M}$ is bounded in $C_{b}(\mathbb{R})$ as well. Now it follows immediately from Corollary 2.3(ii) and Theorem 2.1 that acting conditions and boundedness are equivalent. Indeed, under the conditions of Theorem 2.1, the following assertions are equivalent:

(i) $T_{f}$ satisfies the acting condition $T_{f}\left(B_{p, q}^{s}(\mathbb{R})\right) \subset B_{p, q}^{s}(\mathbb{R})$.

(ii) $T_{f}$ maps bounded sets in $B_{p, q}^{s}(\mathbb{R})$ into bounded sets in $B_{p, q}^{s}(\mathbb{R})$.

(iii) $f \in B_{p, q}^{s, \ell o c}(\mathbb{R})$ and $f(0)=0$.

Remark 2.5. The study of the case $p>q$ requires supplementary conditions involving the parameter $q$. We will prove the above results under the following general conditions: $1<p<+\infty, 1 \leq q \leq+\infty, s>1+(1 / p)$ and

$$
\begin{aligned}
& \text { either: }[s] \geq 1, \quad s-[s]>(1 / p), \quad \frac{p}{q}+\frac{1}{p}-1 \leq s-[s], \\
& \text { or: }[s] \geq 2, \quad s-[s] \leq(1 / p), \quad \frac{p}{q}+\frac{1}{p}-2 \leq s-[s], \\
& \text { or: }[s] \geq 2, \quad 0<s-[s] \leq(1 / p), \quad \frac{2}{p}-1 \leq s-[s] .
\end{aligned}
$$

Notice that $(2.3),(2.4)$ and $(2.5)$ are reduced to the condition $s>1+(1 / p)$ when $p \leq q$.

Remark 2.6. Let $1 \leq p, q \leq+\infty$ and $s>1+(1 / p)$. For any function $f: \mathbb{R} \rightarrow \mathbb{R}$ the question is to prove that the operator $T_{f}$ is bounded on $B_{p, q}^{s}(\mathbb{R})$ if and only if $f(0)=0$ and $f \in B_{p, q}^{s, \ell o c}(\mathbb{R})$. The present article gives an almost complete answer to this question. 
A comment. In [7], under conditions (2.3) or

$$
[s] \geq 2, \quad s-[s] \leq(1 / p), \quad \frac{p}{q}+\frac{1}{p}-2 \leq s-[s], \quad s-[s] \notin J_{p},
$$

where $J_{p}$ denotes the following intervals

$$
J_{p}:= \begin{cases}{\left[\frac{1}{p}-\frac{1}{2}-\sqrt{\frac{1}{p}-\frac{3}{4}}, \frac{1}{p}-\frac{1}{2}+\sqrt{\frac{1}{p}-\frac{3}{4}}\right]} & \text { if } 1<p \leq \frac{4}{3}, \\ \emptyset & \text { if } \quad p>\frac{4}{3},\end{cases}
$$

it has been proved that

$$
\|f \circ g\|_{B_{p, q}^{s}(\mathbb{R})} \leq c\left\|f^{\prime}\right\|_{B_{p, q}^{s-1}(\mathbb{R})}\left(\|g\|_{B_{p, q}^{s}(\mathbb{R})}+\|g\|_{B_{p, q}^{s}(\mathbb{R})}^{s-(1 / p)}\right),
$$

for all $g \in B_{p, q}^{s}(\mathbb{R})$, and all functions $f: \mathbb{R} \rightarrow \mathbb{R}$ such that

$$
f^{\prime} \in B_{p, q}^{s-1}(\mathbb{R}), \quad f(0)=f^{\prime}(0)=\cdots=f^{(m)}(0)=0
$$

for $m:=-[(1 / p)-s]-2$. By Theorem 2.2, in case $m \geq 1$, we weaken the third condition in (2.6) and eliminate the restriction $s-[s] \notin J_{p}$. Also, we do not need the assumption

$$
f^{\prime}(0)=\cdots=f^{(m)}(0)=0 .
$$

The basic idea is to replace condition $f^{\prime} \in B_{p, q}^{s-1}(\mathbb{R})$ by a weaker one, namely $f^{\prime} \in \mathcal{B}_{p, q}^{s-1}(\mathbb{R})$.

\section{The Besov spaces}

First we introduce some further notations. We denote by $\mathcal{P}_{\infty}\left(\mathbb{R}^{n}\right)$ the set of all polynomials on $\mathbb{R}^{n}$, and by $\mathcal{S}_{\infty}\left(\mathbb{R}^{n}\right)$ the set of all $u \in \mathcal{S}\left(\mathbb{R}^{n}\right)$ such that $\langle f, u\rangle=0$ for all $f \in \mathcal{P}_{\infty}\left(\mathbb{R}^{n}\right)$. For all $f \in \mathcal{S}^{\prime}\left(\mathbb{R}^{n}\right)$, we denote by $[f]$ the equivalence class of $f$ modulo $\mathcal{P}_{\infty}\left(\mathbb{R}^{n}\right)$. The mapping which takes any $[f]$ to the restriction of $f$ to $\mathcal{S}_{\infty}\left(\mathbb{R}^{n}\right)$ turns out to be a vector space isomorphism from $\mathcal{S}^{\prime}\left(\mathbb{R}^{n}\right) / \mathcal{P}_{\infty}\left(\mathbb{R}^{n}\right)$ onto $\mathcal{S}_{\infty}^{\prime}\left(\mathbb{R}^{n}\right)$. For this reason, $\mathcal{S}_{\infty}^{\prime}\left(\mathbb{R}^{n}\right)$ is called the space of distributions modulo polynomials.

\subsection{The Littlewood-Paley setting}

We consider the cut-off function $\rho$, see (1.1). We define

$$
\gamma(\xi):=\rho(\xi)-\rho(2 \xi), \quad \forall \xi \in \mathbb{R}^{n} .
$$

Then $\gamma$ is supported in the compact annulus $1 / 2 \leq|\xi| \leq 3 / 2$, and the following identities hold:

$$
\begin{gathered}
\sum_{j \in \mathbb{Z}} \gamma\left(2^{j} \xi\right)=1, \quad \forall \xi \in \mathbb{R}^{n} \backslash\{0\}, \\
\rho(\xi)+\sum_{j \geq 1} \gamma\left(2^{-j} \xi\right)=1, \quad \forall \xi \in \mathbb{R}^{n} .
\end{gathered}
$$


For any $j \in \mathbb{Z}$, we introduce the pseudodifferential operators $S_{j}:=\rho\left(2^{-j} D\right)$ and $Q_{j}:=\gamma\left(2^{-j} D\right)$. It is clear that $S_{j}$ is defined on $\mathcal{S}^{\prime}\left(\mathbb{R}^{n}\right)$ and that $Q_{j}$ is defined on $\mathcal{S}_{\infty}^{\prime}\left(\mathbb{R}^{n}\right)$. All these operators take values in the space of analytical functions of exponential type, see the Paley-Wiener Theorem. The LittlewoodPaley decompositions of a tempered distribution are described in the following well known statements:

Proposition 3.1. (i) For every $f \in \mathcal{S}_{\infty}\left(\mathbb{R}^{n}\right)$ (resp. $\left.\mathcal{S}_{\infty}^{\prime}\left(\mathbb{R}^{n}\right)\right)$, it holds that

$$
f=\sum_{j \in \mathbb{Z}} Q_{j} f
$$

in $\mathcal{S}_{\infty}\left(\mathbb{R}^{n}\right)\left(\right.$ resp. $\left.\mathcal{S}_{\infty}^{\prime}\left(\mathbb{R}^{n}\right)\right)$.

(ii) For every $f \in \mathcal{S}\left(\mathbb{R}^{n}\right)$ (resp. $\mathcal{S}^{\prime}\left(\mathbb{R}^{n}\right)$ ) and every $k \in \mathbb{Z}$, it holds that

$$
f=S_{k} f+\sum_{j>k} Q_{j} f,
$$

in $\mathcal{S}\left(\mathbb{R}^{n}\right)\left(\operatorname{resp} . \mathcal{S}^{\prime}\left(\mathbb{R}^{n}\right)\right)$.

Remark 3.2. According to Young's inequality in $L_{p}\left(\mathbb{R}^{n}\right)$, the families of operators $\left\{S_{j}\right\}_{j \in \mathbb{Z}}$ and $\left\{Q_{j}\right\}_{j \in \mathbb{Z}}$ constitute bounded subsets of the normed space $\mathcal{L}\left(L_{p}\left(\mathbb{R}^{n}\right)\right)$, for any $p \in[1,+\infty]$.

\subsection{Basic facts on homogeneous Besov spaces}

\subsubsection{Definition and some properties.}

Definition 3.3. Let $s \in \mathbb{R}$ and $p, q \in[1, \infty]$. The homogeneous Besov space $\dot{B}_{p, q}^{s}\left(\mathbb{R}^{n}\right)$ is the set of distributions modulo polynomials $f$ such that

$$
\|f\|_{\dot{B}_{p, q}^{s}\left(\mathbb{R}^{n}\right)}:=\left(\sum_{j \in \mathbb{Z}}\left(2^{s j}\left\|Q_{j} f\right\|_{p}\right)^{q}\right)^{1 / q}<+\infty .
$$

Proposition 3.4. Let $a, b$ be real numbers such that $0<a<b$. Let $\left(u_{j}\right)_{j \in \mathbb{Z}}$ be $a$ sequence in $\mathcal{S}^{\prime}\left(\mathbb{R}^{n}\right)$ such that $\operatorname{supp} \widehat{u_{j}} \subseteq\left\{\xi: a 2^{j} \leq|\xi| \leq b 2^{j}\right\}$ and

$$
A:=\left(\sum_{j \in \mathbb{Z}}\left(2^{j s}\left\|u_{j}\right\|_{p}\right)^{q}\right)^{1 / q}<+\infty .
$$

(i) The series $\sum_{j \in \mathbb{Z}} u_{j}$ converges in $\mathcal{S}_{\infty}^{\prime}\left(\mathbb{R}^{n}\right)$, and satisfies

$$
\left\|\sum_{j \in \mathbb{Z}} u_{j}\right\|_{\dot{B}_{p, q}^{s}\left(\mathbb{R}^{n}\right)} \leq c A
$$

where $c$ depends only on $n, s, p, q, a$ and $b$.

(ii) In the case $s>0$, the same conclusion holds for $a=0$; when $s<0$, the same conclusion holds for $b=+\infty$. 
We will exploit the following lemma, a classical consequence of Taylor's formula.

Lemma 3.5. (i) If $f \in \mathcal{S}\left(\mathbb{R}^{n}\right)$, then $\left\|Q_{j} f\right\|_{p}=O\left(2^{-j N}\right)$ as $j \rightarrow+\infty$, for all $N \in \mathbb{N}$. (ii) If $f \in \mathcal{S}_{\infty}\left(\mathbb{R}^{n}\right)$, then $\left\|S_{j} f\right\|_{p}$ and $\left\|Q_{j} f\right\|_{p}$ are $O\left(2^{j N}\right)$ as $j \rightarrow-\infty$, for all $N \in \mathbb{N}$. Proof of Proposition 3.4.

Step 1: Convergence in $\mathcal{S}_{\infty}^{\prime}\left(\mathbb{R}^{n}\right)$. Let $f \in \mathcal{S}_{\infty}\left(\mathbb{R}^{n}\right)$.

Substep 1.1: The case $s>0$. We assume that $\widehat{u_{j}}$ is supported by the ball $|\xi| \leq b 2^{j}$. There exists an integer $m$, depending only on $b$, such that $S_{j+m}\left(u_{j}\right)=u_{j}$, hence $\left\langle u_{j}, f\right\rangle=\left\langle u_{j}, S_{j+m} f\right\rangle$, for all $j \in \mathbb{Z}$. By Lemma 3.5,

$$
\left\|S_{j+m} f\right\|_{p^{\prime}} \leq c(f) \min \left(1,2^{j N}\right), \quad \forall j \in \mathbb{Z},
$$

where $N$ is arbitrarily large. By assumption and by Hölder's inequality, we obtain

$$
\sum_{j \in \mathbb{Z}}\left|\left\langle u_{j}, f\right\rangle\right| \leq c_{1}(f) A
$$

Substep 1.2: The case $s<0$. Now we assume that $\widehat{u_{j}}$ is supported by the set $|\xi| \geq a 2^{j}$. There exists an integer $m$, depending only on $a$, such that $S_{j+m}\left(u_{j}\right)=0$, hence $\left\langle u_{j}, f\right\rangle=\left\langle u_{j}, f-S_{j+m} f\right\rangle$, for all $j \in \mathbb{Z}$. By Lemma 3.5 and Proposition 3.1, we deduce

$$
\left\|S_{j+m} f-f\right\|_{p^{\prime}} \leq c(f) \min \left(1,2^{-j N}\right), \quad \forall j \in \mathbb{Z},
$$

where $N$ is arbitrarily large. We obtain (3.4) as in Substep 1.1.

Substep 1.3: The case $s=0$. There exist integers $m_{1}, m_{2}$, depending only on $a$ and $b$, such that $Q_{k}\left(u_{j}\right)=0$ except perhaps for $m_{1}<j-k<m_{2}$. Then

$$
\left\langle u_{j}, f\right\rangle=\sum_{m_{1}<m<m_{2}}\left\langle u_{j}, Q_{j-m} f\right\rangle .
$$

We conclude using Lemma 3.5, as in the preceding substeps.

Step 2: We turn to prove estimate (3.3). Let $u:=\sum_{j \in \mathbb{Z}} u_{j}$. By Step 1, we have

$$
Q_{k} u=\sum_{k+m_{1}<j<k+m_{2}} Q_{k}\left(u_{j}\right) .
$$

where $m_{1} \in \mathbb{Z} \cup\{-\infty\}, m_{1}=-\infty$ iff $b=+\infty, m_{2} \in \mathbb{Z} \cup\{+\infty\}, m_{2}=+\infty$ iff $a=0$. By Remark 3.2,

$$
2^{k s}\left\|Q_{k} u\right\|_{p} \leq c \sum_{k+m_{1}<j<k+m_{2}} 2^{(k-j) s} 2^{j s}\left\|u_{j}\right\|_{p} .
$$

In all cases, we have

$$
B:=\sum_{m_{1}<l<m_{2}} 2^{-l s}<+\infty .
$$

Then, applying Young's inequality in $l_{q}(\mathbb{Z})$, we obtain

$$
\left(\sum_{k \in \mathbb{Z}}\left(2^{k s}\left\|Q_{k} u\right\|_{p}\right)^{q}\right)^{1 / q} \leq c A B .
$$


Remark 3.6. Proposition 3.4 is a variant of the classical Nikol'skij representation method, see Proposition 2.3.2 (1), page 59, of [16], or [19].

Theorem 3.7. Let $s \in \mathbb{R}$ and $p, q \in[1, \infty]$. $\dot{B}_{p, q}^{s}\left(\mathbb{R}^{n}\right)$ is a Banach space, and the following chain of continuous embeddings holds:

$$
\mathcal{S}_{\infty}\left(\mathbb{R}^{n}\right) \hookrightarrow \dot{B}_{p, q}^{s}\left(\mathbb{R}^{n}\right) \hookrightarrow \mathcal{S}_{\infty}^{\prime}\left(\mathbb{R}^{n}\right)
$$

The above properties are easy consequences of Proposition 3.4, Lemma 3.5, and the completeness of vector valued $L_{p}$ spaces. Using again Proposition 3.4, we derive the following three propositions. The first one explains why our spaces are called homogeneous.

Proposition 3.8. Let $s \in \mathbb{R}$ and $p, q \in[1, \infty]$. Then there exist constants $c_{1}, c_{2}>0$ such that

$$
c_{1}\|f\|_{\dot{B}_{p, q}^{s}\left(\mathbb{R}^{n}\right)} \leq \lambda^{(n / p)-s}\|f(\lambda(\cdot))\|_{\dot{B}_{p, q}^{s}\left(\mathbb{R}^{n}\right)} \leq c_{2}\|f\|_{\dot{B}_{p, q}^{s}\left(\mathbb{R}^{n}\right)}
$$

for all $f \in \dot{B}_{p, q}^{s}\left(\mathbb{R}^{n}\right)$ and all $\lambda>0$.

Remark 3.9. It follows immediately from Proposition 3.8 that we can renorm $\dot{B}_{p, q}^{s}\left(\mathbb{R}^{n}\right)$ in such a way that $c_{1}=c_{2}=1$ in $(3.5)$.

Proposition 3.10. Let $s \in \mathbb{R}$ and $p, q \in[1, \infty]$. A member $f$ of $\mathcal{S}_{\infty}^{\prime}\left(\mathbb{R}^{n}\right)$ belongs to $\dot{B}_{p, q}^{s}\left(\mathbb{R}^{n}\right)$ if and only if its first order derivatives $\partial_{\ell} f$ belongs to $\dot{B}_{p, q}^{s-1}\left(\mathbb{R}^{n}\right)$, $(\ell=1, \ldots, n)$. Moreover $\sum_{\ell=1}^{n}\left\|\partial_{\ell} f\right\|_{\dot{B}_{p, q}^{s-1}\left(\mathbb{R}^{n}\right)}$ is an equivalent norm in $\dot{B}_{p, q}^{s}\left(\mathbb{R}^{n}\right)$.

Proposition 3.11. The continuous embedding $\dot{B}_{p_{1}, q}^{s_{1}}\left(\mathbb{R}^{n}\right) \hookrightarrow \dot{B}_{p_{2}, q}^{s_{2}}\left(\mathbb{R}^{n}\right)$ holds for all parameters $s_{1}, s_{2} \in \mathbb{R}$ and $p_{1}, p_{2}, q \in[1, \infty]$ such that

$$
s_{1}-\frac{n}{p_{1}}=s_{2}-\frac{n}{p_{2}} \quad \text { and } \quad p_{2} \geq p_{1} .
$$

3.2.2. Duality. We start by introducing the following notations. For $p \in[1, \infty]$, we denote by $p^{\prime}$ its conjugate exponent, that is, $(1 / p)+\left(1 / p^{\prime}\right)=1$. If $E$ is any vector space, we denote by $c_{c}(\mathbb{Z}, E)$ the set of sequences $\left(u_{j}\right)_{j \in \mathbb{Z}} \in E^{\mathbb{Z}}$ such that $u_{j}=0$, except for a finite number of indexes $j$, and by $c_{0}(\mathbb{Z}, E)$ the set of sequences $\left(u_{j}\right)_{j \in \mathbb{Z}} \in E^{\mathbb{Z}}$ such that $\lim _{|j| \rightarrow+\infty}\left\|u_{j}\right\|_{E}=0$.

Any homogeneous Besov space is the dual space of a separable Banach space. More precisely, we have the following:

Theorem 3.12. Let $s \in \mathbb{R}, 1<p<+\infty$ and $1 \leq q \leq+\infty$. Then the homogeneous Besov space $\dot{B}_{p, q}^{s}\left(\mathbb{R}^{n}\right)$ is the set of $f \in \mathcal{S}_{\infty}^{\prime}\left(\mathbb{R}^{n}\right)$ such that

$$
N_{p, q}^{s}(f):=\sup \left\{|\langle f, g\rangle|: g \in \mathcal{S}_{\infty}\left(\mathbb{R}^{n}\right),\|g\|_{\dot{B}_{p^{\prime}, q^{\prime}}^{-s}\left(\mathbb{R}^{n}\right)} \leq 1\right\}<+\infty .
$$

Moreover $N_{p, q}^{s}$ is an equivalent norm in $\dot{B}_{p, q}^{s}\left(\mathbb{R}^{n}\right)$. Consequently $\dot{B}_{p, q}^{s}\left(\mathbb{R}^{n}\right)$ can be identified as the dual space of the closure of $\mathcal{S}_{\infty}\left(\mathbb{R}^{n}\right)$ in $\dot{B}_{p^{\prime}, q^{\prime}}^{-s}\left(\mathbb{R}^{n}\right)$. 
Proof. Although the proof is essentially the same as for inhomogeneous Besov spaces (see, for instance, $\S 2.11$ in Triebel's [17]), we outline it below for the convenience of the reader.

We introduce a radial function $\tilde{\gamma} \in \mathcal{D}\left(\mathbb{R}^{n} \backslash\{0\}\right)$ such that $\tilde{\gamma} \gamma=\gamma$, and we define $\widetilde{Q_{j}}:=\tilde{\gamma}\left(2^{-j} D\right)$. Then we can define a duality bracket between $\dot{B}_{p, q}^{s}\left(\mathbb{R}^{n}\right)$ and $\dot{B}_{p^{\prime}, q^{\prime}}^{-s}\left(\mathbb{R}^{n}\right)$ by means of the following:

$$
\triangleleft f, g \triangleright:=\sum_{j \in \mathbb{Z}}\left\langle Q_{j} f, \widetilde{Q_{j}} g\right\rangle .
$$

Using Propositions 3.1 and 3.4, we obtain that

$$
\begin{gathered}
\triangleleft f, g \triangleright=\langle f, g\rangle, \quad \forall f \in \dot{B}_{p, q}^{s}\left(\mathbb{R}^{n}\right), \forall g \in \mathcal{S}_{\infty}\left(\mathbb{R}^{n}\right), \\
|\triangleleft f, g \triangleright| \leq c\|f\|_{\dot{B}_{p, q}^{s}\left(\mathbb{R}^{n}\right)}\|g\|_{\dot{B}_{p^{\prime}, q^{\prime}}^{-s}\left(\mathbb{R}^{n}\right)}, \quad \forall f \in \dot{B}_{p, q}^{s}\left(\mathbb{R}^{n}\right), \forall g \in \dot{B}_{p^{\prime}, q^{\prime}}^{-s}\left(\mathbb{R}^{n}\right) .
\end{gathered}
$$

By the above two relations, we conclude that any $f \in \dot{B}_{p, q}^{s}\left(\mathbb{R}^{n}\right)$ satisfies the prop$\operatorname{erty}(3.6)$, with $N_{p, q}^{s}(f) \leq c\|f\|_{\dot{B}_{p, q}^{s}\left(\mathbb{R}^{n}\right)}$.

Assume conversely that $f$ satisfies the property (3.6). Let use define

$$
\mathcal{L}\left(\left(g_{j}\right)_{j \in \mathbb{Z}}\right):=\left\langle f, \sum_{j \in \mathbb{Z}} 2^{-s j} \widetilde{Q_{j}} g_{j}\right\rangle, \quad \forall\left(g_{j}\right)_{j \in \mathbb{Z}} \in c_{c}\left(\mathbb{Z}, \mathcal{S}_{\infty}\left(\mathbb{R}^{n}\right)\right) .
$$

We set $E=L_{p^{\prime}}\left(\mathbb{R}^{n}\right)$, if $p^{\prime}<\infty$, and $E=C_{0}\left(\mathbb{R}^{n}\right)$, if $p^{\prime}=\infty$. By Proposition 3.4, we see that $\mathcal{L}$ extends as a continuous linear form on $l_{q^{\prime}}(\mathbb{Z}, E)$ if $q^{\prime}<\infty$, and on $c_{0}(\mathbb{Z}, E)$ if $q^{\prime}=\infty$, and that $\|\mathcal{L}\| \leq c_{1} N_{p, q}^{s}(f)$ in the respective dual spaces. By classical duality properties of vector valued $L_{p}$ spaces, there exists a sequence $\left(f_{j}\right)_{j \in \mathbb{Z}}$ such that $\left\|\left(f_{j}\right)_{j \in \mathbb{Z}}\right\|_{l_{q}\left(\mathbb{Z}, E^{\prime}\right)} \leq c_{1} N_{p, q}^{s}(f)$ and

$$
\mathcal{L}\left(\left(g_{j}\right)_{j \in \mathbb{Z}}\right)=\sum_{j \in \mathbb{Z}}\left\langle f_{j}, g_{j}\right\rangle, \quad \forall\left(g_{j}\right)_{j \in \mathbb{Z}} \in c_{c}\left(\mathbb{Z}, \mathcal{S}_{\infty}\left(\mathbb{R}^{n}\right)\right) .
$$

The above identity implies that

$$
f=\sum_{j \in \mathbb{Z}} 2^{-s j} Q_{j} f_{j}
$$

Then Proposition 3.4 and Remark 3.2 give us

$$
\|f\|_{\dot{B}_{p, q}^{s}\left(\mathbb{R}^{n}\right)} \leq c_{2}\left(\sum_{j \in \mathbb{Z}}\left\|Q_{j} f_{j}\right\|_{p}^{q}\right)^{1 / q} \leq c_{3} N_{p, q}^{s}(f)
$$

and the proof is finished.

Next we turn to the Fatou property:

Proposition 3.13. Let $s \in \mathbb{R}$ and $p, q \in[1, \infty]$. Let $f \in \mathcal{S}_{\infty}^{\prime}\left(\mathbb{R}^{n}\right)$. Let $\left(u_{k}\right)_{k \geq 0}$ be a bounded sequence in $\dot{B}_{p, q}^{s}\left(\mathbb{R}^{n}\right)$ such that $\lim _{k \rightarrow+\infty} u_{k}=f$ in $\mathcal{S}_{\infty}^{\prime}\left(\mathbb{R}^{n}\right)$. Then $f \in \dot{B}_{p, q}^{s}\left(\mathbb{R}^{n}\right)$ and

$$
\|f\|_{\dot{B}_{p, q}^{s}\left(\mathbb{R}^{n}\right)} \leq c \liminf _{k \rightarrow+\infty}\left\|u_{k}\right\|_{\dot{B}_{p, q}^{s}\left(\mathbb{R}^{n}\right)} .
$$


Proof. If $1<p<+\infty$, the assertion follows immediately from Theorem 3.12. In the cases $p=1$ and $p=+\infty$, the proof given by Franke [10] for the case of inhomogeneous Besov spaces can be easily extended to hold for homogeneous spaces (see Proposition 3.18 below).

\subsection{Inhomogeneous Besov spaces}

By using the inhomogeneous Littlewood-Paley decomposition (3.2) instead of the homogeneous one (3.1), we obtain the inhomogeneous, or ordinary, Besov spaces:

Definition 3.14. Let $s \in \mathbb{R}$ and $p, q \in[1, \infty]$. The inhomogeneous Besov space $B_{p, q}^{s}\left(\mathbb{R}^{n}\right)$ is the set of tempered distributions $f$ such that

$$
\|f\|_{B_{p, q}^{s}\left(\mathbb{R}^{n}\right)}:=\left\|S_{0} f\right\|_{p}+\left(\sum_{j \geq 1}\left(2^{s j}\left\|Q_{j} f\right\|_{p}\right)^{q}\right)^{1 / q}<+\infty .
$$

The properties of the homogeneous spaces listed in Subsections 3.2.1 and 3.2.2 have well known counterparts for the inhomogeneous ones, see Triebel [17]. Except for $s=0$, the latter can be easily deduced from the former. Indeed we have the following:

Proposition 3.15. Let $s>0$ and $1 \leq p, q \leq+\infty$. Then it holds

$$
B_{p, q}^{s}\left(\mathbb{R}^{n}\right)=\left\{f \in L_{p}\left(\mathbb{R}^{n}\right):[f] \in \dot{B}_{p, q}^{s}\left(\mathbb{R}^{n}\right)\right\} .
$$

Moreover $\|f\|_{p}+\|f\|_{\dot{B}_{p, q}^{s}\left(\mathbb{R}^{n}\right)}$ is an equivalent norm in $B_{p, q}^{s}\left(\mathbb{R}^{n}\right)$.

For $s<0, B_{p, q}^{s}\left(\mathbb{R}^{n}\right)$ coincides with $L_{p}\left(\mathbb{R}^{n}\right)+\dot{B}_{p, q}^{s}\left(\mathbb{R}^{n}\right)$, as a set, and as a Banach space. By contrast, there is no embedding relations between $\dot{B}_{p, q}^{0}\left(\mathbb{R}^{n}\right)$ and $B_{p, q}^{0}\left(\mathbb{R}^{n}\right)$, except for $p=q=2\left(\right.$ then both coincide with $L_{2}\left(\mathbb{R}^{n}\right)$ ).

3.3.1. Characterization by differences. First, for brevity we will use the following notation. For a measurable function $f$ on $\mathbb{R}^{n}$ we put

$$
M_{p, q}^{s, m, u}(f):=\left(\int_{0}^{\infty} t^{-s q}\left(\int_{\mathbb{R}^{n}}\left(t^{-n} \int_{|h| \leq t}\left|\Delta_{h}^{m} f(x)\right|^{u} \mathrm{~d} h\right)^{p / u} \mathrm{~d} x\right)^{q / p} \frac{\mathrm{d} t}{t}\right)^{1 / q}
$$

Now, according to Triebel (see Theorem 3.5.3, page 194, in [18]), we have an equivalent norm on $B_{p, q}^{s}\left(\mathbb{R}^{n}\right)$ via difference operators at our disposal.

Proposition 3.16. Let $1 \leq p, q, u \leq+\infty$. Let $0<s<m$ for some integer $m \geq 1$. Assume

$$
s>n\left(\frac{1}{p}-\frac{1}{u}\right) .
$$

Then a function $f \in L_{p}\left(\mathbb{R}^{n}\right)$ belongs to $B_{p, q}^{s}\left(\mathbb{R}^{n}\right)$ if and only if $M_{p, q}^{s, m, u}(f)<+\infty$. Moreover, $\|f\|_{p}+M_{p, q}^{s, m}(f)$ is an equivalent norm in $B_{p, q}^{s}\left(\mathbb{R}^{n}\right)$. This assertion remains true if, in the expression of the term $M_{p, q}^{s, m, u}(f)$, one replaces $\int_{0}^{\infty} \ldots \mathrm{d} t$ by $\int_{0}^{a} \ldots \mathrm{d} t$ for any fixed $a>0$. 
Remark 3.17. Using Theorem 2.3.8 of [17], we can replace $s$ and $f$ by $s-k$ and $\partial_{\ell}^{k} f(\ell=1, \ldots, n)$ for some integers $k \geq 0$, respectively, in Proposition 3.16.

\subsubsection{The Fatou property.}

Proposition 3.18. Let $s \in \mathbb{R}$ and $p, q \in[1, \infty]$. Let $\left(u_{k}\right)_{k \geq 0}$ be a bounded sequence in $B_{p, q}^{s}\left(\mathbb{R}^{n}\right)$ converging to $f$ in $\mathcal{S}^{\prime}\left(\mathbb{R}^{n}\right)$. Then $f \in B_{p, q}^{s}\left(\mathbb{R}^{n}\right)$ and

$$
\|f\|_{B_{p, q}^{s}\left(\mathbb{R}^{n}\right)} \leq c \liminf _{k \rightarrow+\infty}\left\|u_{k}\right\|_{B_{p, q}^{s}\left(\mathbb{R}^{n}\right)} .
$$

Proof. See [10].

\subsection{Besov algebras}

3.4.1. Definition and some properties. For $s \in \mathbb{R}$ and $p, q \in[1, \infty]$ we introduce the function space

$$
\mathcal{B}_{p, q}^{s}\left(\mathbb{R}^{n}\right):=\left\{f \in L_{\infty}\left(\mathbb{R}^{n}\right):[f] \in \dot{B}_{p, q}^{s}\left(\mathbb{R}^{n}\right)\right\}
$$

and we endow it with its natural norm, i.e.,

$$
\|f\|_{\mathcal{B}_{p, q}^{s}\left(\mathbb{R}^{n}\right)}:=\|f\|_{\infty}+\|f\|_{\dot{B}_{p, q}^{s}\left(\mathbb{R}^{n}\right)} .
$$

It will turn out that $\mathcal{B}_{p, q}^{s}\left(\mathbb{R}^{n}\right)$ is a Banach algebra for the pointwise product for any $s>0$, see Subsection 3.4.3 below. In that case, we will refer to these spaces as the Besov algebras.

Theorem 3.19. $\mathcal{B}_{p, q}^{s}\left(\mathbb{R}^{n}\right)$ is a Banach space, and the following chain of continuous embeddings holds:

$$
\mathcal{S}_{\infty}\left(\mathbb{R}^{n}\right) \hookrightarrow \mathcal{B}_{p, q}^{s}\left(\mathbb{R}^{n}\right) \hookrightarrow \mathcal{S}^{\prime}\left(\mathbb{R}^{n}\right)
$$

Proof. It follows from Theorem 3.7 and Lemma 3.5.

Remark 3.20. In the case $s>0$, the left hand side embedding in (3.9) can be improved in the sense that $\mathcal{S}\left(\mathbb{R}^{n}\right)$ is embedded into $\mathcal{B}_{p, q}^{s}\left(\mathbb{R}^{n}\right)$. This follows from $B_{p, q}^{s}\left(\mathbb{R}^{n}\right) \hookrightarrow \dot{B}_{p, q}^{s}\left(\mathbb{R}^{n}\right)$.

The following alternative description of certain multi-dimensional Besov algebras is also of interest. We show that Besov algebras can be viewed as substitutes of inhomogeneous Besov spaces, in case the latter are themselves algebras.

Proposition 3.21. Let $1 \leq p, q \leq+\infty$.

(i) For $s>n / p$, we have

$$
B_{p, q}^{s}\left(\mathbb{R}^{n}\right) \hookrightarrow \mathcal{B}_{p, q}^{s}\left(\mathbb{R}^{n}\right)=\dot{B}_{p, q}^{s}\left(\mathbb{R}^{n}\right) \cap C_{u b}\left(\mathbb{R}^{n}\right) \hookrightarrow B_{\infty, q}^{s-(n / p)}\left(\mathbb{R}^{n}\right) .
$$

(ii) For $s>1+(n / p)$, it holds that

$$
\mathcal{B}_{p, q}^{s}\left(\mathbb{R}^{n}\right)=\left\{f \in L_{\infty}\left(\mathbb{R}^{n}\right): \partial_{\ell} f \in \mathcal{B}_{p, q}^{s-1}\left(\mathbb{R}^{n}\right), \ell=1, \ldots, n\right\},
$$

and $\|f\|_{\infty}+\sum_{\ell=1}^{n}\left\|\partial_{\ell} f\right\|_{\mathcal{B}_{p, q}^{s-1}\left(\mathbb{R}^{n}\right)}$ is an equivalent norm in $\mathcal{B}_{p, q}^{s}\left(\mathbb{R}^{n}\right)$. 
Proof. Step 1: Proof of (i). The first embedding follows from Proposition 3.15 and the Sobolev type embedding $B_{p, q}^{s}\left(\mathbb{R}^{n}\right) \hookrightarrow C_{b}\left(\mathbb{R}^{n}\right)$. The second one follows from Proposition 3.11.

By (3.2), any function in $\dot{B}_{p, q}^{s}\left(\mathbb{R}^{n}\right)$ is the sum of a function of $B_{p, q}^{s}\left(\mathbb{R}^{n}\right)$ and an entire function of exponential type. Thus in case $s>n / p, \dot{B}_{p, q}^{s}\left(\mathbb{R}^{n}\right)$ is embedded into $C\left(\mathbb{R}^{n}\right)$.

Step 2: Proof of (ii). By Proposition 3.10, it suffices to establish the inequality

$$
\left\|\partial_{\ell} f\right\|_{\infty} \leq c\|f\|_{\mathcal{B}_{p, q}^{s}\left(\mathbb{R}^{n}\right)}, \quad \forall f \in \mathcal{B}_{p, q}^{s}\left(\mathbb{R}^{n}\right) .
$$

To prove (3.10) we observe, by (i), that $\partial_{\ell} f \in B_{\infty, q}^{s-1-(n / p)}\left(\mathbb{R}^{n}\right)$. Now we use the inequality

$$
\left\|\partial_{\ell} f\right\|_{B_{\infty, q}^{s-1-(n / p)}\left(\mathbb{R}^{n}\right)} \leq c\|f\|_{B_{\infty, q}^{s-(n / p)}\left(\mathbb{R}^{n}\right)},
$$

and we again apply (i). Notice that the last inequality can be easily obtained by means of Bernstein's inequality. We omit the details.

For the following assertion, we need first to introduce the notion of distributions vanishing at the infinity, cf. Definition 6 in [5].

Definition 3.22. A tempered distribution $f \in \mathcal{S}^{\prime}\left(\mathbb{R}^{n}\right)$ is said to vanish at infinity in the weak sense if

$$
\lim _{\lambda \rightarrow 0} f(\dot{\bar{\lambda}})=0 \quad \text { in } \mathcal{S}^{\prime}\left(\mathbb{R}^{n}\right)
$$

Example of such distributions are the functions $f \in C_{0}\left(\mathbb{R}^{n}\right)$, the functions $\partial_{\ell} f$ such that $f \in C_{b}\left(\mathbb{R}^{n}\right)$ and the functions $f \in L_{p}\left(\mathbb{R}^{n}\right)$ for $1 \leq p<+\infty$.

Proposition 3.23. Let $s>\max (1, n / p)$ and $1 \leq p, q<+\infty$. Let $v$ be a real number satisfying

$$
\max \left(\frac{1}{n}\left(1+\frac{n}{p}-s\right), 0\right)<\frac{1}{v}<\frac{1}{s p}
$$

Then there exists a constant $c>0$ such that $\left\|\partial_{\ell} f\right\|_{v} \leq c\|f\|_{\mathcal{B}_{p, q}^{s}\left(\mathbb{R}^{n}\right)}$ for all $f \in$ $\mathcal{B}_{p, q}^{s}\left(\mathbb{R}^{n}\right),(\ell=1, \ldots, n)$.

Proof. Without loss of generality, we can assume that $q=+\infty$. Let us set

$$
f_{1}:=\sum_{j \leq 0} Q_{j} f, \quad f_{2}:=\sum_{j>0} Q_{j} f .
$$

Notice that $f_{2}$ is a distribution, while $f_{1}$ a distribution modulo polynomials. By Proposition 3.4, it holds that $\left\|f_{2}\right\|_{\dot{B}_{p, \infty}^{s}\left(\mathbb{R}^{n}\right)} \leq c\|f\|_{\dot{B}_{p, \infty}^{s}\left(\mathbb{R}^{n}\right)}$.

Since $\left\|Q_{j} f\right\|_{p} \leq c 2^{-s j}\|f\|_{\dot{B}_{p, \infty}^{s}\left(\mathbb{R}^{n}\right)}$, the series $\sum_{j>0} Q_{j} f$ converges in $L_{p}\left(\mathbb{R}^{n}\right)$. By Proposition 3.15 we conclude that

$$
\left\|f_{2}\right\|_{B_{p, \infty}^{s}\left(\mathbb{R}^{n}\right)} \leq c\|f\|_{\dot{B}_{p, \infty}^{s}\left(\mathbb{R}^{n}\right)} .
$$


By the classical Sobolev embedding, $B_{p, \infty}^{s}\left(\mathbb{R}^{n}\right) \hookrightarrow W_{v}^{1}\left(\mathbb{R}^{n}\right)$ if $s-(n / p)>1-(n / v)$ and $p<v$, see, e.g., Corollary 2, page 36 in [16]. Thus we conclude that

$$
\left\|\partial_{\ell} f_{2}\right\|_{v} \leq c\|f\|_{\dot{B}_{p, \infty}^{s}\left(\mathbb{R}^{n}\right)}
$$

under condition (3.11).

We turn to the estimate of $f_{1}$. By Bernstein's inequality, and by the assumption $p<v$, it holds that

$$
\begin{aligned}
\left\|\partial_{\ell}\left(Q_{j} f\right)\right\|_{v} & \leq c_{1} 2^{j}\left\|Q_{j} f\right\|_{v} \\
& \leq c_{1} 2^{j}\left\|Q_{j} f\right\|_{p}^{p / v}\left\|Q_{j} f\right\|_{\infty}^{1-(p / v)} \\
& \leq c_{2} 2^{j(1-(s p / v))}\|f\|_{\dot{B}_{p, \infty}^{s}\left(\mathbb{R}^{n}\right)}^{p / v}\|f\|_{\infty}^{1-(p / v)} .
\end{aligned}
$$

By the assumption $v>s p$, the series $\sum_{j \leq 0} \partial_{\ell}\left(Q_{j} f\right)$ converges in $L_{v}\left(\mathbb{R}^{n}\right)$. If we denote its sum by $g$, then

$$
\|g\|_{v} \leq c\|f\|_{\mathcal{B}_{p, \infty}^{s}\left(\mathbb{R}^{n}\right)}
$$

under condition (3.11).

The assumption $s>n / p$ yields $f \in C_{b}\left(\mathbb{R}^{n}\right)$, see Proposition 3.21(i). By (3.1), we know that $\partial_{\ell} f$ and $g+\partial_{\ell} f_{2}$ differ by a polynomial. Since both tend to 0 at infinity, in the weak sense, we conclude that they coincide. By combining (3.12) and (3.13), we obtain the desired estimate.

Remark 3.24. Proposition 3.23 is also valid in case $p=v=+\infty$. See the proof of (3.10).

3.4.2. Characterization by differences. According to Triebel (see [18], Theorem 3.5.3, page 194), we can describe $\mathcal{B}_{p, q}^{s}\left(\mathbb{R}^{n}\right)$ by difference operators, as the inhomogeneous Besov space, see Subsection 3.3.1. We will use the notation $M_{p, q}^{s, m}$ defined by formula (3.7).

Proposition 3.25. Let $1 \leq p, q, u \leq+\infty$.

(i) Let $0<s<m$, for some integer $m \geq 1$. Assume (3.8). Then there exists a constant $c>0$ such that $M_{p, q}^{s, m, u}(f) \leq c\|f\|_{\dot{B}_{p, q}^{s}\left(\mathbb{R}^{n}\right)}$ holds for all $f \in \mathcal{B}_{p, q}^{s}\left(\mathbb{R}^{n}\right)$.

(ii) Let $\max (1, n / p)<s<m+1$, for some integer $m \geq 1$. Assume (3.8) with $s-1$ instead of $s$. Then there exists a constant $c>0$ such that $M_{p, q}^{s-1, m, u}\left(\partial_{\ell} f\right) \leq$ $c\|f\|_{\dot{B}_{p, q}^{s}\left(\mathbb{R}^{n}\right)}$ holds for all $f \in \mathcal{B}_{p, q}^{s}\left(\mathbb{R}^{n}\right),(\ell=1, \ldots, n)$.

Proof. (i) By Propositions 3.15 and 3.16, it holds that

$$
M_{p, q}^{s, m, u}(f) \leq c\left(\|f\|_{p}+\|f\|_{\dot{B}_{p, q}^{s}\left(\mathbb{R}^{n}\right)}\right), \quad \forall f \in B_{p, q}^{s}\left(\mathbb{R}^{n}\right) .
$$

We replace now $f$ by $f(\lambda(\cdot))$, for any $\lambda>0$, in (3.14). Using Proposition 3.8, dividing by $\lambda^{s-(n / p)}$, and letting $\lambda \rightarrow+\infty$, we obtain

$$
M_{p, q}^{s, m, u}(f) \leq c\|f\|_{\dot{B}_{p, q}^{s}\left(\mathbb{R}^{n}\right)}, \quad \forall f \in B_{p, q}^{s}\left(\mathbb{R}^{n}\right) .
$$


Now we take $f \in \mathcal{B}_{p, q}^{s}\left(\mathbb{R}^{n}\right)$, and we set $g_{k}:=f-S_{-k} f+S_{-k} f(0)$ for $k \geq 0$. Then the sequence $\left(g_{k}\right)_{k \geq 0}$ has the following properties:

- $\left\|g_{k}-S_{-k} f(0)\right\|_{\dot{B}_{p, q}^{s}\left(\mathbb{R}^{n}\right)} \leq c\|f\|_{\dot{B}_{p, q}^{s}\left(\mathbb{R}^{n}\right)}$, see Proposition 3.4.

- $g_{k}-S_{-k} f(0) \in B_{p, q}^{s}\left(\mathbb{R}^{n}\right)$; indeed the assumption $f \in \dot{B}_{p, q}^{s}\left(\mathbb{R}^{n}\right)$ yields $Q_{j} f \in$ $L_{p}\left(\mathbb{R}^{n}\right)$ for all $j \in \mathbb{Z}$, then by Hölder's inequality it holds that

$$
\begin{aligned}
\left\|g_{k}-S_{-k} f(0)\right\|_{p} & \leq \sum_{j=-k+1}^{-1}\left\|Q_{j} f\right\|_{p}+\sum_{j \geq 0} 2^{-s j}\left(2^{s j}\left\|Q_{j} f\right\|_{p}\right) \\
& \leq \sum_{j=-k+1}^{-1}\left\|Q_{j} f\right\|_{p}+c\|f\|_{\dot{B}_{p, q}^{s}\left(\mathbb{R}^{n}\right)} .
\end{aligned}
$$

- $g_{k}$ tends to $f$ pointwise; indeed by Bernstein's inequality, the following holds:

$$
\left|S_{k} f(x)-S_{k} f(0)\right| \leq|x|\left\|\nabla\left(S_{k} f\right)\right\|_{\infty} \leq c 2^{k}|x|\|f\|_{\infty}, \quad \forall x \in \mathbb{R}^{n},
$$

hence $\lim _{k \rightarrow-\infty}\left(S_{k} f-S_{k} f(0)\right)=0$ uniformly on all compact subsets of $\mathbb{R}^{n}$, that implies the desired conclusion.

Then applying (3.15) to $g_{k}-S_{-k} f(0)$, we obtain

$$
M_{p, q}^{s, m, u}\left(g_{k}\right)=M_{p, q}^{s, m, u}\left(g_{k}-S_{-k} f(0)\right) \leq c\|f\|_{\dot{B}_{p, q}^{s}\left(\mathbb{R}^{n}\right)}, \quad \forall k \geq 0 .
$$

By a repeated use of Fatou's Lemma, we deduce the result.

(ii) As in (3.14), combining Propositions 3.15 and 3.16, and Remark 3.17, we obtain

$$
M_{p, q}^{s-1, m, u}\left(\partial_{\ell} f\right) \leq c\left(\|f\|_{p}+\|f\|_{\dot{B}_{p, q}^{s}\left(\mathbb{R}^{n}\right)}\right), \quad \forall f \in B_{p, q}^{s}\left(\mathbb{R}^{n}\right) .
$$

With an elementary changes of variable, we get

$$
M_{p, q}^{s-1, m, u}\left(\partial_{\ell}(f(\lambda \cdot))\right)=\lambda^{s-(n / p)} M_{p, q}^{s-1, m, u}\left(\partial_{\ell} f\right), \quad \forall \lambda>0 .
$$

Replacing $f$ by $f(\lambda \cdot)$ in (3.16), using Proposition 3.8, and again dividing by $\lambda^{s-(n / p)}$ and letting $\lambda \rightarrow+\infty$, we obtain

$$
M_{p, q}^{s-1, m, u}\left(\partial_{\ell} f\right) \leq c\|f\|_{\dot{B}_{p, q}^{s}\left(\mathbb{R}^{n}\right)}, \quad f \in B_{p, q}^{s}\left(\mathbb{R}^{n}\right) .
$$

Now consider any function $f \in \mathcal{B}_{p, q}^{s}\left(\mathbb{R}^{n}\right)$. Let us define

$$
f_{k}:=\sum_{j=-k+1}^{k} Q_{j} f, \quad \forall k \geq 1 .
$$

The assumption $f \in \dot{B}_{p, q}^{s}\left(\mathbb{R}^{n}\right)$ implies $Q_{j} f \in L_{p}\left(\mathbb{R}^{n}\right)$ for all $j$, hence $f_{k} \in L_{p}\left(\mathbb{R}^{n}\right)$. Now, by Proposition 3.4 it holds that

$$
\left\|f_{k}\right\|_{\dot{B}_{p, q}^{s}\left(\mathbb{R}^{n}\right)} \leq c\|f\|_{\dot{B}_{p, q}^{s}\left(\mathbb{R}^{n}\right)}, \quad \forall k \geq 1 .
$$


We deduce that $f_{k} \in B_{p, q}^{s}\left(\mathbb{R}^{n}\right)$. Then combining (3.17) and (3.18), we derive

$$
M_{p, q}^{s-1, m, u}\left(\partial_{\ell} f_{k}\right) \leq c\|f\|_{\dot{B}_{p, q}^{s}\left(\mathbb{R}^{n}\right)}, \quad \forall k \geq 1 .
$$

By definition, it holds that

$$
\partial_{\ell} f_{k}=\sum_{j=-k+1}^{k} Q_{j}\left(\partial_{\ell} f\right), \quad \forall k \geq 1
$$

Let $v$ be a real number satisfy (3.11). It follows, from Proposition 3.23, that $\partial_{\ell} f_{k} \rightarrow \partial_{\ell} f$ in $L_{v}\left(\mathbb{R}^{n}\right)$. Taking a subsequence if necessary, and applying twice Fatou's Lemma in (3.19), we deduce the desired estimate.

3.4.3. The algebra property. As mentioned above, we are now turning to the algebra property of $\mathcal{B}_{p, q}^{s}\left(\mathbb{R}^{n}\right)$.

Theorem 3.26. For $s>0, \mathcal{B}_{p, q}^{s}\left(\mathbb{R}^{n}\right)$ is a unital Banach algebra for the pointwise product.

The main ingredient of the proof is the following paraproduct estimate, a straightforward consequence of Proposition 3.4:

Lemma 3.27. Assume $s>0$ and $m \in \mathbb{Z}$. For all $f \in L_{\infty}\left(\mathbb{R}^{n}\right)$ and all $g \in$ $\dot{B}_{p, q}^{s}\left(\mathbb{R}^{n}\right)$, let us define

$$
\pi_{m}(f, g):=\sum_{j \in \mathbb{Z}}\left(S_{j-m} f\right)\left(Q_{j} g\right)
$$

Then $\pi_{m}$ is a continuous bilinear application from $L_{\infty}\left(\mathbb{R}^{n}\right) \times \dot{B}_{p, q}^{s}\left(\mathbb{R}^{n}\right)$ to $\dot{B}_{p, q}^{s}\left(\mathbb{R}^{n}\right)$.

Proof of Theorem 3.26. Let us take $f, g$ in $\mathcal{B}_{p, q}^{s}\left(\mathbb{R}^{n}\right)$. By an Abel transform, we deduce that

$$
\sum_{k=-j}^{j}\left(S_{k} f\right)\left(Q_{k} g\right)+\sum_{k=-j}^{j-1}\left(S_{k} g\right)\left(Q_{k+1} f\right)=\left(S_{j} f\right)\left(S_{j} g\right)-\left(S_{-j} f\right)\left(S_{-j-1} g\right),
$$

for all $j>0$. We claim that

$$
\lim _{j \rightarrow-\infty}\left(S_{j} f\right)\left(S_{j-1} g\right)=0 \quad \text { in } \quad \mathcal{S}_{\infty}^{\prime}\left(\mathbb{R}^{n}\right) .
$$

Indeed, for $u \in \mathcal{S}_{\infty}\left(\mathbb{R}^{n}\right)$, we have

$$
\left\langle\left(S_{j} f\right)\left(S_{j-1} g\right), u\right\rangle=\left\langle\left(S_{j} f\right)\left(S_{j-1} g\right), S_{j+2} u\right\rangle .
$$

Then our claim follows from Lemma 3.5 and Remark 3.2. On the other hand, we have classically $\left|S_{j} f(x)\right| \leq\|f\|_{\infty}$, for all $x \in \mathbb{R}^{n}$ and $j \in \mathbb{Z}$, and $S_{j} f(x) \rightarrow f(x)$ as $j \rightarrow+\infty$ for almost every $x \in \mathbb{R}^{n}$. By the Dominated Convergence Theorem, we deduce that

$$
\lim _{j \rightarrow+\infty}\left(S_{j} f\right)\left(S_{j} g\right)=f g \quad \text { in } \quad \mathcal{S}^{\prime}\left(\mathbb{R}^{n}\right) .
$$


Now taking $j \rightarrow+\infty$ in $(3.20)$, we obtain

$$
\pi_{0}(f, g)+\pi_{1}(g, f)=[f g] \quad \text { in } \quad \mathcal{S}_{\infty}^{\prime}\left(\mathbb{R}^{n}\right) .
$$

Using Lemma 3.27, we conclude that

$$
\|[f g]\|_{\dot{B}_{p, q}^{s}\left(\mathbb{R}^{n}\right)} \leq c\left(\|f\|_{\infty}\|g\|_{\dot{B}_{p, q}^{s}\left(\mathbb{R}^{n}\right)}+\|g\|_{\infty}\|f\|_{\dot{B}_{p, q}^{s}\left(\mathbb{R}^{n}\right)}\right),
$$

the desired estimate.

\section{Proof of Theorem 2.2}

\subsection{Some reduction arguments}

Theorem 2.2 can be obtained from the following assertion: The estimate (2.2) holds for all parameters $s, p, q$ and all functions $f$ and $g$ satisfying the following conditions:

(i) $1<p<+\infty, 1 \leq q \leq+\infty, 1+(1 / p)<s \leq 2+(1 / p)$, and either $(2.3)$ or $(2.4)$ or $(2.5)$;

(ii) $f$ is of class $C^{2}, f^{\prime} \in \mathcal{B}_{p, q}^{s-1}$ and $f(0)=0$;

(iii) $g$ is real analytic and $g \in B_{p, q}^{s}$.

Step 1: Let us prove inequality (2.2) for all functions $f$ and $g$ such that $f^{\prime} \in$ $\mathcal{B}_{p, q}^{s-1}, f(0)=0$ and $g \in B_{p, q}^{s}$.

We use the operators $S_{j}$ introduced in Subsection 3.1. Then $g_{j}:=S_{j} g$ is real analytic, $g_{j} \rightarrow g$ in $L_{p}$, and by translation invariance of $B_{p, q}^{s}$, it holds that

$$
\left\|g_{j}\right\|_{B_{p, q}^{s}} \leq c\|g\|_{B_{p, q}^{s}}, \quad \forall j \geq 0
$$

Applying the embedding $\mathcal{B}_{p, q}^{s-1} \hookrightarrow C_{u b}$, see Proposition 3.21(i), and some standard computations, we obtain that

$$
\lim _{j \rightarrow+\infty}\left\|\left(S_{j} f\right)^{\prime}-f^{\prime}\right\|_{\infty}=0 \quad \text { and } \quad\left\|S_{j} f-f\right\|_{\infty} \leq c 2^{-j}\left\|f^{\prime}\right\|_{\infty}
$$

Let us define $f_{j}:=S_{j} f-S_{j} f(0) \rho$, see (1.1) for the definition of function $\rho$. Then $f_{j}$ is a $C^{\infty}$ function such that $f_{j}(0)=0$ and

$$
\left\|f_{j}^{\prime}\right\|_{\mathcal{B}_{p, q}^{s-1}} \leq c\left(\left\|f^{\prime}\right\|_{\mathcal{B}_{p, q}^{s-1}}+\left|S_{j} f(0)\right|\right) .
$$

Since $\lim _{j \rightarrow+\infty} S_{j} f(0)=f(0)=0$, the estimate (4.2) implies that

$$
\lim _{j \rightarrow+\infty}\left\|f_{j}^{\prime}-f^{\prime}\right\|_{\infty}=0
$$


Now suppose that the inequality (2.2) is satisfied under the assumptions (i), (ii) and (iii). Then using (4.1) and (4.3), we obtain

$$
\left\|f_{j} \circ g_{j}\right\|_{B_{p, q}^{s}} \leq c\left(\left\|f^{\prime}\right\|_{\mathcal{B}_{p, q}^{s-1}}+\left|S_{j} f(0)\right|\right)\left(\|g\|_{B_{p, q}^{s}}+\|g\|_{B_{p, q}^{s}}^{s-(1 / p)}\right) .
$$

The elementary inequality

$$
\left\|f \circ g-f_{j} \circ g_{j}\right\|_{p} \leq\left\|f^{\prime}\right\|_{\infty}\left\|g-g_{j}\right\|_{p}+c\left\|f^{\prime}-f_{j}^{\prime}\right\|_{\infty}\|g\|_{p}, \quad \forall j \geq 0,
$$

complemented by (4.4), yields the convergence of the sequence $\left(f_{j} \circ g_{j}\right)_{j \geq 0}$ in $L_{p}$. Hence, we can apply the Fatou property in (4.5) (see Proposition 3.18). Using again $\lim _{j \rightarrow+\infty} S_{j} f(0)=0$, we conclude that the inequality (2.2) holds.

Step 2: Let us prove the inequality (2.1) for all functions $f$ and $g$ such that $f^{\prime} \in \mathcal{B}_{p, q}^{s-1}, f(0)=0$ and $g \in B_{p, q}^{s}$.

Indeed, by the embedding $B_{p, q}^{s-1} \hookrightarrow C_{b}$, Propositions 3.10 and 3.15, and Step 1, we find

$$
\begin{aligned}
\left\|(f \circ g)^{\prime}\right\|_{\mathcal{B}_{p, q}^{s-1}} & =\left\|(f \circ g)^{\prime}\right\|_{\infty}+\left\|(f \circ g)^{\prime}\right\|_{\dot{B}_{p, q}^{s-1}} \\
& \leq\left\|f^{\prime}\right\|_{\infty}\left\|g^{\prime}\right\|_{\infty}+c_{1}\|f \circ g\|_{B_{p, q}^{s}} \\
& \leq c_{2}\left\|f^{\prime}\right\|_{\mathcal{B}_{p, q}^{s-1}}\left(\|g\|_{B_{p, q}^{s}}+\|g\|_{B_{p, q}^{s}}^{s-(1 / p)}\right) .
\end{aligned}
$$

Step 3: Since $(f \circ g)^{\prime}=((f-f(0)) \circ g)^{\prime}$, then the inequality $(2.1)$ holds in the general case.

Step 4: Argument of the restriction on the parameters. We assume that (2.1) holds under the restriction (i), and prove it for general $s$ by induction on $[s]$.

Assume that $f^{\prime} \in \mathcal{B}_{p, q}^{s}$ and $g \in B_{p, q}^{s+1}$. Then using Theorem 3.26, the induction assumption, Proposition 3.21(ii), Remark 3.17 and the embeddings $B_{p, q}^{s+1} \hookrightarrow$ $B_{p, q}^{s} \hookrightarrow \mathcal{B}_{p, q}^{s}$, we deduce

$$
\begin{aligned}
\left\|(f \circ g)^{\prime}\right\|_{\mathcal{B}_{p, q}^{s}} & \leq c_{1}\left\|f^{\prime} \circ g\right\|_{\mathcal{B}_{p, q}^{s}}\left\|g^{\prime}\right\|_{\mathcal{B}_{p, q}^{s}} \\
& \leq c_{2}\left(\left\|f^{\prime} \circ g\right\|_{\infty}+\left\|\left(f^{\prime} \circ g\right)^{\prime}\right\|_{\mathcal{B}_{p, q}^{s-1}}\right)\|g\|_{B_{p, q}^{s+1}} \\
& \leq c_{3}\left(\left\|f^{\prime}\right\|_{\infty}+\left\|f^{\prime \prime}\right\|_{\mathcal{B}_{p, q}^{s-1}}\left(\|g\|_{B_{p, q}^{s}}+\|g\|_{B_{p, q}^{s}}^{s-(1 / p)}\right)\right)\|g\|_{B_{p, q}^{s+1}} \\
& \leq c_{4}\left\|f^{\prime}\right\|_{\mathcal{B}_{p, q}^{s}}\left(\|g\|_{B_{p, q}^{s+1}}+\|g\|_{B_{p, q}^{s+1}}^{s+1-(1 / p)}\right) .
\end{aligned}
$$

The last inequality follows from $\|g\|_{B_{p, q}^{s+1}}^{2} \leq\|g\|_{B_{p, q}^{s+1}}+\|g\|_{B_{p, q}^{s+1}}^{s+1-(1 / p)}$.

\subsection{Details of the proof}

First, we begin with some notations. For all measurable function $f$ on $\mathbb{R}^{n}$, all $m \in \mathbb{N} \backslash\{0\}$ and all $t>0$, we set

$$
\Omega_{p}^{m}(f ; t):=\left(\int_{\mathbb{R}} \sup _{|h| \leq t}\left|\Delta_{h}^{m} f(x)\right|^{p} \mathrm{~d} x\right)^{1 / p} .
$$


The Hardy-Littlewood maximal function $M g$ of a locally integrable function $g$ is defined as

$$
M g(x):=\sup \frac{1}{|I|} \int_{I}|g(y)| \mathrm{d} y, \quad x \in \mathbb{R},
$$

where the supremum is taken with respect to all intervals $I$ containing $x$. Here $|I|$ means the length of the interval $I$.

For any function $g: \mathbb{R} \rightarrow \mathbb{R}$, we denote by $\nu_{p}(g)$ the supremum of numbers

$$
\left(\sum_{k=1}^{N}\left|g\left(b_{k}\right)-g\left(a_{k}\right)\right|^{p}\right)^{1 / p}
$$

taken over all finite sets \{]$a_{k}, b_{k}[; k=1, \ldots, N\}$ of pairwise disjoint open intervals. If $\nu_{p}(g)<+\infty$, we say that $g$ is a function of bounded $p$-variation. This notion was first introduced by N. Wiener [20]. The set of (generalized) primitives of real functions of bounded $p$-variation is denoted by $B V_{p}^{1}\left(=B V_{p}^{1}(\mathbb{R})\right)$ and endowed with the seminorm $\|g\|_{B V_{p}^{1}}$ defined as the infimum of numbers $\nu_{p}(f)$, for all functions $f$ whose $g$ is the primitive, see [4] for further details. We will apply in particular the Peetre embedding theorem:

$$
B_{p, 1}^{1+(1 / p)} \hookrightarrow B V_{p}^{1}, \quad 1 \leq p<+\infty ;
$$

see Theorem 7, page 112 in [14] or Theorem 5 in [4].

Now under the assumptions (i), (ii) and (iii) of Subsection 4.1 we will prove the estimate (2.2). For simplicity we define

$$
V(f ; g):=\left\|f^{\prime}\right\|_{\mathcal{B}_{p, q}^{s-1}}\left(\|g\|_{B_{p, q}^{s}}+\|g\|_{B_{p, q}^{s}}^{s-(1 / p)}\right) .
$$

The argument of our proof will be distributed into three subsections, corresponding to the following cases:
(a) $1+(1 / p)<s<2$,
(b) $s=2$,
(c) $2<s \leq 2+(1 / p)$.

In the cases (a) and (b), the proof is essentially given in [6] and [7]. But we need to replace the right hand side of $(2.7)$ by $V(f ; g)$. We will use the equivalent norms given by Proposition 3.16 and Remark 3.17. We will first estimate $\|f \circ g\|_{p}$, but from the assumption $f(0)=0$ we will obtain the bound $\left\|f^{\prime}\right\|_{\infty}\|g\|_{p}$ which is bounded by $c V(f ; g)$. Secondly, we will estimate $M_{p, q}^{s-1,1, \infty}\left((f \circ g)^{\prime}\right), M_{p, q}^{1,2, \infty}\left((f \circ g)^{\prime}\right)$ and $M_{p, q}^{s-2,1,1}\left((f \circ g)^{\prime \prime}\right)$ in the above cases (a), (b) and (c), respectively; see (3.7) for the definition of $M_{p, q}^{s, m, u}$.

4.2.1. The case $1+(1 / p)<s<2$. We will prove the estimate

$$
A:=\left(\int_{0}^{\infty}\left(\frac{\Omega_{p}^{1}\left((f \circ g)^{\prime} ; t\right)}{t^{s-1}}\right)^{q} \frac{\mathrm{d} t}{t}\right)^{1 / q} \leq c V(f ; g) .
$$

We have

$$
\Omega_{p}^{1}\left(\left(f^{\prime} \circ g\right) g^{\prime} ; t\right) \leq\left\|f^{\prime}\right\|_{\infty} \Omega_{p}^{1}\left(g^{\prime} ; t\right)+U(t)
$$


where

$$
U(t):=\left(\int_{\mathbb{R}}\left|g^{\prime}(x)\right|^{p} \sup _{|h| \leq t}\left|\Delta_{h}\left(f^{\prime} \circ g\right)(x)\right|^{p} \mathrm{~d} x\right)^{1 / p} .
$$

Thus we are reduced to prove $(4.7)$ with $\Omega_{p}^{1}\left((f \circ g)^{\prime} ; t\right)$ replaced by $U(t)$.

Step 1: Assume that $g^{\prime}$ does not vanish. By the Mean Value Theorem and with a change of variable we find that

$$
\begin{aligned}
U(t) & \leq\left\|g^{\prime}\right\|_{\infty}^{1-(1 / p)}\left(\int_{\mathbb{R}}\left|g^{\prime}(x)\right| \sup _{|h| \leq t}\left|\Delta_{h}\left(f^{\prime} \circ g\right)(x)\right|^{p} \mathrm{~d} x\right)^{1 / p} \\
& \leq\left\|g^{\prime}\right\|_{\infty}^{1-(1 / p)} \Omega_{p}^{1}\left(f^{\prime} ;\left\|g^{\prime}\right\|_{\infty} t\right) .
\end{aligned}
$$

With a change of variable with respect to $t$, we obtain

$$
\left(\int_{0}^{\infty}\left(\frac{U(t)}{t^{s-1}}\right)^{q} \frac{\mathrm{d} t}{t}\right)^{1 / q} \leq c M_{p, q}^{s-1,1, \infty}\left(f^{\prime}\right)\left\|g^{\prime}\right\|_{\infty}^{s-(1 / p)} .
$$

Hence, the desired estimate holds from Proposition 3.25(i)

Step 2: Assume that the set of zeros of $g^{\prime}$ is nonempty. Then it is a discrete set, whose complement in $\mathbb{R}$ is the union of a family $\left(I_{l}\right)$ of open disjoint intervals. For any $t>0$ we denote by $I_{l, t}^{\prime}$ the set of $x \in I_{l}$ whose distance to the boundary of $I_{l}$ is greater than $2 t$, and we set

$$
I_{l, t}^{\prime \prime}:=I_{l} \backslash I_{l, t}^{\prime}, \quad a_{l}:=\sup _{I_{l}}\left|g^{\prime}\right| .
$$

Let us notice that $I_{l, t}^{\prime}$ is an open interval, possibly empty. In case it is not empty, we have

$$
\left|g\left(g_{\mid I_{l}}^{-1}(y)+h\right)-g\left(g_{\mid I_{l}}^{-1}(y)\right)\right| \leq a_{l} t, \quad \forall y \in g\left(I_{l, t}^{\prime}\right), \quad \forall h \in[-t, t] .
$$

The set $I_{l, t}^{\prime \prime}$ is an interval of length at most $2 t$, or the union of two such intervals, and $g^{\prime}$ vanishes at one of the endpoints of this or those intervals. Now we introduce

$$
U_{1}(t):=\left(\sum_{l} \int_{I_{l, t}^{\prime}}\left|g^{\prime}(x)\right|^{p} \sup _{|h| \leq t}\left|\Delta_{h}\left(f^{\prime} \circ g\right)(x)\right|^{p} \mathrm{~d} x\right)^{1 / p} .
$$

$U_{2}(t)$ is defined in the same way, by replacing $I_{l, t}^{\prime}$ by $I_{l, t}^{\prime \prime}$. $A_{1}$ and $A_{2}$ are defined accordingly, by replacing, in $(4.7), \Omega_{p}^{1}\left((f \circ g)^{\prime} ; t\right)$ by $U_{1}(t)$ and $U_{2}(t)$ respectively.

Estimate of $A_{1}$. With the change of variable $y:=g_{\mid I_{l}}(x)$, and using (4.9), we deduce that

$$
\begin{aligned}
\int_{I_{l, t}^{\prime}}\left|g^{\prime}(x)\right|^{p} \sup _{|h| \leq t} & \left|\Delta_{h}\left(f^{\prime} \circ g\right)(x)\right|^{p} \mathrm{~d} x \leq \\
& \leq a_{l}^{p-1} \int_{g\left(I_{l, t}^{\prime}\right)} \sup _{|h| \leq t}\left|f^{\prime}\left(g\left(g_{\mid I_{l}}^{-1}(y)+h\right)\right)-f^{\prime}(y)\right|^{p} \mathrm{~d} y \\
& \leq a_{l}^{p-1}\left(\Omega_{p}^{1}\left(f^{\prime} ; a_{l} t\right)\right)^{p}, \quad \forall h \in[-t, t] .
\end{aligned}
$$


We put $\alpha:=\min (1, q / p)$. Then it is easy to obtain the following estimate:

$$
\sum_{l} \int_{I_{l, t}^{\prime}}\left|g^{\prime}(x)\right|^{p} \sup _{|h| \leq t}\left|\Delta_{h}\left(f^{\prime} \circ g\right)(x)\right|^{p} \mathrm{~d} x \leq\left(\sum_{l} a_{l}^{\alpha(p-1)}\left(\Omega_{p}^{1}\left(f^{\prime} ; a_{l} t\right)\right)^{\alpha p}\right)^{1 / \alpha}
$$

By Minkowski's inequality w.r.t $L_{q /(\alpha p)}\left(\mathbb{R}^{+}, \mathrm{d} t / t\right)$, and by a change of variable w.r.t. $t$, we obtain

$$
A_{1} \leq c M_{p, q}^{s-1,1, \infty}\left(f^{\prime}\right)\left(\sum_{l} a_{l}^{\alpha(s p-1)}\right)^{1 /(\alpha p)} .
$$

Now by Proposition 3.25(i) and the fact that $g^{\prime}$ vanishes at the endpoints of $I_{l}$, we deduce that

$$
A_{1} \leq c\left\|f^{\prime}\right\|_{\mathcal{B}_{p, q}^{s-1}}\|g\|_{B V_{\alpha(s p-1)}^{1}}^{s-(1 / p)} .
$$

Estimate of $A_{2}$. By the properties of $I_{l, t}^{\prime \prime}$, it holds that

$$
\left|g^{\prime}(x)\right| \leq \sup _{|h| \leq 2 t}\left|g^{\prime}(x)-g^{\prime}(x+h)\right|, \quad \forall x \in I_{l, t}^{\prime \prime} .
$$

The elementary inequality $\left|\Delta_{h}\left(f^{\prime} \circ g\right)(x)\right| \leq 2\left\|f^{\prime}\right\|_{\infty}$ implies that

$$
U_{2}(t) \leq 2\left\|f^{\prime}\right\|_{\infty}\left(\int_{\mathbb{R}} \sup _{|h| \leq 2 t}\left|g^{\prime}(x)-g^{\prime}(x+h)\right|^{p} \mathrm{~d} x\right)^{1 / p} \leq 2\left\|f^{\prime}\right\|_{\infty} \Omega_{p}^{1}\left(g^{\prime} ; 2 t\right) .
$$

Hence, by Remark 3.17,

$$
A_{2} \leq c_{1}\left\|f^{\prime}\right\|_{\infty}\left\|g^{\prime}\right\|_{B_{p, q}^{s-1}} \leq c_{2}\left\|f^{\prime}\right\|_{\infty}\|g\|_{B_{p, q}^{s}} .
$$

Combining inequalities (4.12) and (4.14), we obtain that

$$
\|f \circ g\|_{B_{p, q}^{s}} \leq c\left\|f^{\prime}\right\|_{\mathcal{B}_{p, q}^{s-1}}\left(\|g\|_{B_{p, q}^{s}}+\|g\|_{B V_{\alpha(s p-1)}^{s-(1 / p)}}^{1}\right) .
$$

The inequalities $s>1+(1 / p)$ and $p \leq \alpha(s p-1)$, which are equivalent to $(2.3)$, allow us to deduce the embeddings:

$$
B_{p, q}^{s} \hookrightarrow B_{\alpha(s p-1), 1}^{1+\frac{1}{\alpha(s p-1)}} \hookrightarrow B V_{\alpha(s p-1)}^{1} \quad(\text { see }(4.6)) .
$$

The desired estimate follows from (4.15) and (4.16).

4.2.2. The case $s=2$. Instead of the second order difference operator $\Delta_{h}^{2}$, we will use its symmetric counterpart, namely we put $\widetilde{\Delta}_{h}^{2} f(x):=\Delta_{h}^{2} f(x-h)$. Then we can write

$$
\widetilde{\Delta}_{h}^{2}\left(\left(f^{\prime} \circ g\right) g^{\prime}\right)(x)=A_{1}(x, h)+A_{2}(x, h)+\frac{1}{2} \sum_{j=3}^{5}\left(A_{j}(x, h)+A_{j}(x,-h)\right),
$$


where the $A_{j}$ 's are defined by:

$$
\begin{aligned}
& A_{1}(x, h):=f^{\prime}(g(x+h)) \widetilde{\Delta}_{h}^{2}\left(g^{\prime}\right)(x), \\
& A_{2}(x, h):=\left(g^{\prime}(x)-g^{\prime}(x-h)\right)\left(f^{\prime}(g(x+h))-f^{\prime}(g(x-h))\right), \\
& A_{3}(x, h):=g^{\prime}(x)\left(f^{\prime}(g(x+h))+f^{\prime}(2 g(x)-g(x+h))-2 f^{\prime}(g(x))\right), \\
& A_{4}(x, h):=\left(g^{\prime}(x)-g^{\prime}(x+h)\right)\left(f^{\prime}(g(x+h))-f^{\prime}(2 g(x)-g(x-h))\right), \\
& A_{5}(x, h):=g^{\prime}(x+h)\left(f^{\prime}(g(x+h))-f^{\prime}(2 g(x)-g(x-h))\right) .
\end{aligned}
$$

It suffices to prove

$$
U_{j}:=\left(\int_{0}^{1} t^{-q}\left(\int_{\mathbb{R}} \sup _{|h| \leq t}\left|A_{j}(x, h)\right|^{p} \mathrm{~d} x\right)^{q / p} \frac{\mathrm{d} t}{t}\right)^{1 / q} \leq c V(f ; g)
$$

for $j=1, \ldots, 5$. In most cases, the above estimate will follow from

$$
\left(\int_{\mathbb{R}} \sup _{|h| \leq t}\left|A_{j}(x, h)\right|^{p} \mathrm{~d} x\right)^{1 / p} \leq c t^{\alpha} V(f ; g) \quad \text { for some } \alpha>1 .
$$

Estimate of $U_{1}$. This is obvious since $f^{\prime} \in L_{\infty}$.

Estimate of $U_{2}$. Using the embeddings

$$
\mathcal{B}_{p, q}^{1} \hookrightarrow B_{\infty, \infty}^{1-(1 / p)}, \quad B_{p, q}^{1} \hookrightarrow B_{p, \infty}^{\gamma} \quad(\text { for } \quad 0<\gamma<1),
$$

see Propositions 3.11 and $3.21(\mathrm{i})$, we derive

$$
\left(\int_{\mathbb{R}|h| \leq t} \sup _{\mid A_{2}}\left|A_{2}(x)\right|^{p} \mathrm{~d} x\right)^{1 / p} \leq c\left(t\left\|g^{\prime}\right\|_{\infty}\right)^{1-(1 / p)}\left\|f^{\prime}\right\|_{\mathcal{B}_{p, q}^{1}} \Omega_{p}^{1}\left(g^{\prime} ; t\right) .
$$

Choose $1 / p<\gamma<1$ (which follows by $p>1$ ), we obtain, in the right hand side of (4.20), the bound

$$
c t^{1-(1 / p)+\gamma}\|g\|_{B_{p, q}^{2}}^{1-(1 / p)}\left\|g^{\prime}\right\|_{B_{p, \infty}^{\gamma}}\left\|f^{\prime}\right\|_{\mathcal{B}_{p, q}^{1}} .
$$

We conclude that (4.18) holds for $j=2$.

Estimate of $U_{3}$. As in Step 1 of Subsection 4.2.1, we first assume that $g^{\prime}(x) \neq 0$ for all $x \in \mathbb{R}$. Then we obtain

$$
\begin{aligned}
\left(\int_{\mathbb{R}} \sup _{|h| \leq t}\left|A_{3}(x, h)\right|^{p} \mathrm{~d} x\right)^{1 / p} & \leq\left\|g^{\prime}\right\|_{\infty}^{1-(1 / p)}\left(\int_{\mathbb{R}}\left|g^{\prime}(x)\right|_{|u| \leq t\left\|g^{\prime}\right\|_{\infty}}\left|\left(\widetilde{\Delta}_{u}^{2} f^{\prime}\right)(g(x))\right|^{p} \mathrm{~d} x\right)^{1 / p} \\
& \leq\left\|g^{\prime}\right\|_{\infty}^{1-(1 / p)} \Omega_{p}^{2}\left(f^{\prime} ; t\left\|g^{\prime}\right\|_{\infty}\right) .
\end{aligned}
$$

The estimate (4.17), for $j=3$, follows by the change of variable $v:=\left\|g^{\prime}\right\|_{\infty} t$ with respect to $t$ and Proposition 3.25(i). 
We turn now to the general case. We use the notation $I_{l}, I_{l, t}^{\prime}, I_{l, t}^{\prime \prime}$ and $a_{l}$ of (4.8). Then $U_{3} \leq U_{3}^{\prime}+U_{3}^{\prime \prime}$, where

$$
U_{3}^{\prime}:=\left(\int_{0}^{1} t^{-q}\left(\sum_{l} \int_{I_{l, t}^{\prime}} \sup _{|h| \leq t}\left|A_{3}(x, h)\right|^{p} \mathrm{~d} x\right)^{q / p} \frac{\mathrm{d} t}{t}\right)^{1 / q},
$$

and $U_{3}^{\prime \prime}$ is similar to $U_{3}^{\prime}$, with $I_{l, t}^{\prime \prime}$ instead of $I_{l, t}^{\prime}$. To estimate $U_{3}^{\prime}$, we argue as in estimate of $A_{1}$ of Subsection 4.2.1, see in particular (4.9). It holds that

$$
\int_{I_{l, t}^{\prime}} \sup _{|h| \leq t}\left|A_{3}(x, h)\right|^{p} \mathrm{~d} x \leq a_{l}^{p-1}\left(\Omega_{p}^{2}\left(f^{\prime} ; a_{l} t\right)\right)^{p} .
$$

With the help of Minkowski's inequality, the change of variable $v:=a_{l} t$ (see (4.10) and (4.11)), and Proposition 3.25(i), we obtain that

$$
U_{3}^{\prime} \leq c_{1} M_{p, q}^{1,2, \infty}\left(f^{\prime}\right)\left(\sum_{l} a_{l}^{\alpha(2 p-1)}\right)^{1 /(\alpha p)} \leq c_{2}\left\|f^{\prime}\right\|_{\mathcal{B}_{p, q}^{1}}\|g\|_{B V_{\alpha(2 p-1)}^{1}}^{2-(1 / p)},
$$

where $\alpha=\min (1, q / p)$. The rest can be completed as in (4.16), see also (2.4) for the third condition.

Now we treat the estimate of $U_{3}^{\prime \prime}$. Let us observe that

$$
\sup _{|h| \leq t}\left|f^{\prime}(g(x+h))+f^{\prime}(2 g(x)-g(x+h))-2 f^{\prime}(g(x))\right| \leq \Omega_{\infty}^{2}\left(f^{\prime} ; t\left\|g^{\prime}\right\|_{\infty}\right) .
$$

Using the fact that $\left|I_{l, t}^{\prime \prime}\right| \leq 2 t$, and the estimate

$$
\left(\sum_{l} \sup _{I_{l, t}^{\prime \prime}}\left|g^{\prime}\right|^{p}\right)^{1 / p} \leq\|g\|_{B V_{p}^{1}} \leq c\|g\|_{B_{p, q}^{2}}
$$

we get that

$$
\begin{aligned}
U_{3}^{\prime \prime} & \leq c_{1}\left(\sum_{l} \sup _{I_{l, t}^{\prime \prime}}\left|g^{\prime}\right|^{p}\right)^{1 / p}\left(\int_{0}^{\infty}\left(\frac{\Omega_{\infty}^{2}\left(f^{\prime} ; t\left\|g^{\prime}\right\|_{\infty}\right)}{t^{1-(1 / p)}}\right)^{q} \frac{\mathrm{d} t}{t}\right)^{1 / q} \\
& \leq c_{2} M_{\infty, q}^{1-(1 / p), 2, \infty}\left(f^{\prime}\right)\|g\|_{B_{p, q}^{2}}\left\|g^{\prime}\right\|_{\infty}^{1-(1 / p)} .
\end{aligned}
$$

The last inequality is obtained by the change of variable $v:=\left\|g^{\prime}\right\|_{\infty} t$ with respect to $t$. We conclude by Proposition $3.25(\mathrm{i})$ and the embedding $\mathcal{B}_{p, q}^{1} \hookrightarrow \dot{B}_{\infty, q}^{1-(1 / p)}$.

Estimate of $U_{4}$. This can be done as the estimate of $U_{2}$.

Estimate of $U_{5}$. This is the more delicate part of the present subsection. We consider a parameter $v>p$. By Hölder's inequality, it holds that

$$
\left(\int_{\mathbb{R}} \sup _{|h| \leq t}\left|A_{5}(x, h)\right|^{p} \mathrm{~d} x\right)^{1 / p} \leq\left\|g^{\prime}\right\|_{p}^{1-(p / v)}\left(\int_{\mathbb{R}} \sup _{|h| \leq t} A_{6}(x, h) \mathrm{d} x\right)^{1 / v},
$$

where

$$
A_{6}(x, h):=\left|g^{\prime}(x)\right|^{p}\left|f^{\prime}(g(x))-f^{\prime}(2 g(x-h)-g(x-2 h))\right|^{v} .
$$


It holds that

$$
U_{5} \leq\left\|g^{\prime}\right\|_{p}^{1-(p / v)}\left(U_{5}^{\prime}+U_{5}^{\prime \prime}\right)
$$

where

$$
U_{5}^{\prime}:=\left(\int_{0}^{1} t^{-q}\left(\sum_{l} \int_{I_{l, t}^{\prime}} \sup _{|h| \leq t} A_{6}(x, h) \mathrm{d} x\right)^{q / v} \frac{\mathrm{d} t}{t}\right)^{1 / q},
$$

and $U_{5}^{\prime \prime}$ is defined similarly, by replacing $I_{l, t}^{\prime}$ by $I_{l, t}^{\prime \prime}$.

For brevity, we put $\beta:=1-(1 / p)$, and we begin with the estimate of $U_{5}^{\prime}$. Clearly for every $x \in I_{l, t}^{\prime}$, it holds that

$$
\sup _{|h| \leq t}|g(x-2 h)+g(x)-2 g(x-h)| \leq c \min \left(t a_{l}, t^{\beta+1}\|g\|_{B_{\infty, \infty}^{\beta+1}}\right) .
$$

Then, by the change of variable $y:=g_{\mid I_{l}}(x)$, we have

$$
\int_{I_{l, t}^{\prime}|h| \leq t} \sup _{|h|} A_{6}(x, h) \mathrm{d} x \leq c a_{l}^{p-1}\left\{\Omega_{v}^{1}\left(f^{\prime} ; c \min \left(t a_{l}, t^{\beta+1}\|g\|_{B_{\infty}^{\beta+\infty}}^{\beta+1}\right)\right)\right\}^{v} .
$$

Now we require the following condition on $v$ :

$$
\frac{1}{v}<1-\beta
$$

(Let us notice that (4.22) implies $v>p$ ). Then, the embedding $\mathcal{B}_{p, q}^{1} \hookrightarrow \mathcal{B}_{v, \infty}^{\beta+(1 / v)}$ implies that

$$
\Omega_{v}^{1}\left(f^{\prime} ; z\right) \leq c\left\|f^{\prime}\right\|_{\mathcal{B}_{p, q}^{1}} z^{\beta+(1 / v)}, \quad \forall z>0 .
$$

We put $A:=\|g\|_{B_{\infty, \infty}^{\beta+1}}$. By (4.23) we conclude

$$
\begin{aligned}
& \sum_{l} a_{l}^{p-1}\left\{\Omega_{v}^{1}\left(f^{\prime} ; c_{1} \min \left(t a_{l}, t^{\beta+1} A\right)\right)\right\}^{v} \leq \\
& \leq c_{2}\left\|f^{\prime}\right\|_{\mathcal{B}_{p, q}^{1}}^{v}\left(\sum_{l:\left(a_{l} \leq t^{\beta} A\right)} a_{l}^{p-1}\left(t a_{l}\right)^{\beta v+1}+\sum_{l:\left(a_{l}>t^{\beta} A\right)} a_{l}^{p-1}\left(t^{\beta+1} A\right)^{\beta v+1}\right) \\
& \quad \leq c_{2}\left\|f^{\prime}\right\|_{\mathcal{B}_{p, q}^{1}}^{v}\left(\sum_{l} a_{l}^{p}\right)\left(t^{\beta v+1}\left(t^{\beta} A\right)^{\beta v}+t^{(\beta+1)(\beta v+1)} A^{\beta v+1}\left(t^{-\beta} A^{-1}\right)\right) .
\end{aligned}
$$

From (4.21) and the inequalities

$$
\left(\sum_{l} a_{l}^{p}\right)^{1 / p} \leq c_{1}\|g\|_{B V_{p}^{1}} \leq c_{2}\|g\|_{B_{p, q}^{2}}
$$

we deduce that

$$
\sum_{l} \int_{I_{l, t}^{\prime}} \sup _{|h| \leq t} A_{6}(x, h) \mathrm{d} x \leq c\left\|f^{\prime}\right\|_{\mathcal{B}_{p, q}^{1}}^{v}\|g\|_{B_{p, q}^{2}}^{\beta v+p} t^{\left(\beta^{2}+\beta\right) v+1} .
$$


Concerning the estimate of $U_{5}^{\prime \prime}$, we will proceed as for $U_{3}^{\prime \prime}$. Using embeddings (4.19) and $B_{p, q}^{2} \hookrightarrow B_{\infty, \infty}^{\beta+1}$, we deduce that

$$
\sup _{|h| \leq t}\left|f^{\prime}(g(x))-f^{\prime}(2 g(x-h)-g(x-2 h))\right| \leq c\left\|f^{\prime}\right\|_{\mathcal{B}_{p, q}^{1}}\|g\|_{B_{p, q}^{2}}^{\beta} t^{\beta^{2}+\beta} .
$$

By (4.24) and by $\left|I_{l, t}^{\prime \prime}\right| \leq 2 t$, it holds that

$$
\sum_{l} \int_{I_{l, t}^{\prime \prime}} \sup _{|h| \leq t} A_{6}(x, h) \mathrm{d} x \leq c\left\|f^{\prime}\right\|_{\mathcal{B}_{p, q}^{1}}^{v}\|g\|_{B_{p, q}^{2}}^{\beta v+p} t^{\left(\beta^{2}+\beta\right) v+1} .
$$

By (4.25) and (4.26), we can conclude that (4.18) holds, for $j=5$, if $v$ satisfies

$$
\beta^{2}+\beta+\frac{1}{v}>1
$$

Finally, the property $0<\beta<1$ leads to the realization of (4.22) and (4.27) simultaneously.

4.2.3. The case $2<\boldsymbol{s} \leq \mathbf{2}+(\mathbf{1} / \boldsymbol{p})$. First, we put $\delta:=s-1-(1 / p)-\varepsilon$, with $\varepsilon=0$ if $s<2+(1 / p)$ and $\varepsilon>0$ if $s=2+(1 / p)$. In case $\varepsilon>0$, we take it as small as we want. The parameter $\delta$ plays an important role in our computation.

Since $f$ and $g$ are functions of class $C^{2}$, it holds that

$$
(f \circ g)^{\prime \prime}=\left(f^{\prime \prime} \circ g\right) g^{\prime 2}+\left(f^{\prime} \circ g\right) g^{\prime \prime} .
$$

Step 1: Estimate of $\left(f^{\prime} \circ g\right) g^{\prime \prime}$. By Proposition 3.21(i), $\mathcal{B}_{p, q}^{s-1}$ is embedded into $B_{\infty, \infty}^{\delta}$. Also, $B_{\infty, \infty}^{\delta}$ is the space of bounded Hölder continuous functions of order $\delta$ (recall $0<\delta<1$ ). A straightforward computation leads to

$$
\left\|f^{\prime} \circ g\right\|_{B_{\infty, \infty}^{\delta}} \leq c\left\|f^{\prime}\right\|_{B_{\infty, \infty}^{\delta}}\left(1+\left\|g^{\prime}\right\|_{\infty}^{\delta}\right) .
$$

By a classical result on multipliers, see Theorem 4.7.1 of [16], and by assumption $\delta>s-2$, we deduce that

$$
\left\|\left(f^{\prime} \circ g\right) g^{\prime \prime}\right\|_{B_{p, q}^{s-2}} \leq c\left\|f^{\prime} \circ g\right\|_{B_{\infty, \infty}^{\delta}}\left\|g^{\prime \prime}\right\|_{B_{p, q}^{s-2}} .
$$

Inequalities (4.28) and (4.29) give the desired estimate.

Step 2: Estimate of $\left(f^{\prime \prime} \circ g\right) g^{\prime 2}$. Since $0<s-2<1$, see $(3.8)$, we have to estimate

$$
U:=\left(\int_{0}^{\infty} t^{-(s-2) q}\left(\int_{\mathbb{R}}\left(t^{-1} \int_{-t}^{t}\left|\Delta_{h}\left(\left(f^{\prime \prime} \circ g\right) g^{\prime 2}\right)(x)\right| \mathrm{d} h\right)^{p} \mathrm{~d} x\right)^{q / p} \frac{\mathrm{d} t}{t}\right)^{1 / q}
$$

by $c V(f ; g)$. We split the area of integration with respect to $h$. For $x \in \mathbb{R}$, we define

$$
\begin{aligned}
& Q(x):=\left\{h \in \mathbb{R}:\left|g^{\prime}(x+h)\right| \leq\left|g^{\prime}(x)\right|\right\} \\
& P(x):=\left\{h \in \mathbb{R}:\left|g^{\prime}(x)\right|<\left|g^{\prime}(x+h)\right|\right\} .
\end{aligned}
$$


We put

$$
Q(x ; t):=Q(x) \cap[-t, t] \text { and } P(x ; t):=P(x) \cap[-t, t] .
$$

On $Q(x ; t)$ we will use the elementary identity

$$
\Delta_{h}\left(\left(f^{\prime \prime} \circ g\right) g^{\prime 2}\right)(x)=g^{\prime}(x+h)^{2} \Delta_{h}\left(f^{\prime \prime} \circ g\right)(x)+f^{\prime \prime}(g(x)) \Delta_{h}\left(g^{\prime 2}\right)(x),
$$

whereas on $P(x ; t)$ we will use

$$
\Delta_{h}\left(\left(f^{\prime \prime} \circ g\right) g^{\prime 2}\right)(x)=g^{\prime}(x)^{2} \Delta_{h}\left(f^{\prime \prime} \circ g\right)(x)+f^{\prime \prime}(g(x+h)) \Delta_{h}\left(g^{\prime 2}\right)(x)
$$

instead. Hence $U \leq U_{1}+U_{2}+U_{3}+U_{4}$, where

$$
\begin{aligned}
U_{1} & :=\left(\int_{0}^{\infty} t^{-(s-2) q}\left(\int_{\mathbb{R}}\left(t^{-1} \int_{Q(x ; t)}\left|f^{\prime \prime}(g(x)) \| \Delta_{h}\left(g^{\prime 2}\right)(x)\right| \mathrm{d} h\right)^{p} \mathrm{~d} x\right)^{q / p} \frac{\mathrm{d} t}{t}\right)^{1 / q}, \\
U_{2} & :=\left(\int_{0}^{\infty} t^{-(s-2) q}\left(\int_{\mathbb{R}}\left(t^{-1} \int_{P(x ; t)}\left|f^{\prime \prime}(g(x+h)) \| \Delta_{h}\left(g^{\prime 2}\right)(x)\right| \mathrm{d} h\right)^{p} \mathrm{~d} x\right)^{q / p} \frac{\mathrm{d} t}{t}\right)^{1 / q}, \\
U_{3} & :=\left(\int_{0}^{\infty} t^{-(s-2) q}\left(\int_{\mathbb{R}}\left(t^{-1} \int_{Q(x ; t)}\left|\Delta_{h}\left(f^{\prime \prime} \circ g\right)(x)\right| g^{\prime}(x+h)^{2} \mathrm{~d} h\right)^{p} \mathrm{~d} x\right)^{q / p} \frac{\mathrm{d} t}{t}\right)^{1 / q}, \\
U_{4} & :=\left(\int_{0}^{\infty} t^{-(s-2) q}\left(\int_{\mathbb{R}}\left(t^{-1} \int_{P(x ; t)}\left|\Delta_{h}\left(f^{\prime \prime} \circ g\right)(x)\right| g^{\prime}(x)^{2} \mathrm{~d} h\right)^{p} \mathrm{~d} x\right)^{q / p} \frac{\mathrm{d} t}{t}\right)^{1 / q} .
\end{aligned}
$$

Substep 2.1: Estimate of $U_{3}$. Using the notation $I_{l}, I_{l, t}^{\prime}, I_{l, t}^{\prime \prime}$ and $a_{l}$ of Step 2 in Subsection 4.2.1, see also (4.8) and (4.9), we write

$$
U_{3} \leq U_{3}^{\prime}+U_{3}^{\prime \prime}
$$

where

$U_{3}^{\prime}:=\left(\int_{0}^{\infty} t^{-(s-2) q}\left(\sum_{l} \int_{I_{l, t}^{\prime}}\left(t^{-1} \int_{Q(x ; t)}\left|\Delta_{h}\left(f^{\prime \prime} \circ g\right)(x)\right| g^{\prime}(x+h)^{2} \mathrm{~d} h\right)^{p} \mathrm{~d} x\right)^{q / p} \frac{\mathrm{d} t}{t}\right)^{1 / q}$, and $U_{3}^{\prime \prime}$ is defined in the same way with $I_{l, t}^{\prime \prime}$ instead of $I_{l, t}^{\prime}$.

Estimate of $U_{3}^{\prime}$. By the assumptions on $s$ (see (2.4) and (2.5)), it follows that $s \geq \frac{p}{q}+\frac{1}{p}$ or $s>1+\frac{2}{p}$. Then we consider the following three cases:

- The case $q / p \geq 1$. We begin with the elementary inequality

$$
g^{\prime}(x+h)^{2} \leq\left|g^{\prime}(x)\right|\left|g^{\prime}(x+h)\right|, \quad \forall h \in Q(x),
$$

and we introduce the following changes of variables:

$$
y:=g_{\mid I_{l}}(x) \text { and } \quad \Theta:=\Theta(h)=g\left(g_{\mid I_{l}}^{-1}(y)+h\right)-y \text {. }
$$


Since $|\Theta| \leq a_{l} t$ for all $h \in[-t, t]$, (see (4.9)), we obtain that

$$
\begin{aligned}
\int_{I_{l, t}^{\prime}}\left(t^{-1}\right. & \left.\int_{Q(x ; t)}\left|\Delta_{h}\left(f^{\prime \prime} \circ g\right)(x)\right| g^{\prime}(x+h)^{2} \mathrm{~d} h\right)^{p} \mathrm{~d} x \leq \\
\leq & a_{l}^{p-1} \int_{g\left(I_{l, t}^{\prime}\right)}\left(t^{-1} \int_{Q\left(g_{\mid I_{l}}^{-1}(y) ; t\right)}\right. \\
& \left.\times\left|f^{\prime \prime}\left(g\left(g_{\mid I_{l}}^{-1}(y)+h\right)\right)-f^{\prime \prime}(y)\right|\left|g^{\prime}\left(g_{\mid I_{l}}^{-1}(y)+h\right)\right| \mathrm{d} h\right)^{p} \mathrm{~d} y \\
\leq & a_{l}^{p-1} \int_{\mathbb{R}}\left(t^{-1} \int_{|\Theta| \leq a_{l} t}\left|\Delta_{\Theta} f^{\prime \prime}(y)\right| \mathrm{d} \Theta\right)^{p} \mathrm{~d} y .
\end{aligned}
$$

We apply Minkowski's inequality, the change of variable $v:=a_{l} t$, and Proposition 3.25 (ii) to get

$$
\begin{aligned}
U_{3}^{\prime} & \leq\left(\sum_{l} a_{l}^{p-1}\left\{\int_{0}^{\infty} t^{-(s-2) q}\left(\int_{\mathbb{R}}\left(t^{-1} \int_{|\Theta| \leq a_{l} t}\left|\Delta_{\Theta} f^{\prime \prime}(y)\right| \mathrm{d} \Theta\right)^{p} \mathrm{~d} y\right)^{q / p} \frac{\mathrm{d} t}{t}\right\}^{p / q}\right)^{1 / p} \\
& \leq\left(\sum_{l} a_{l}^{s p-1}\right)^{1 / p}\left(\int_{0}^{\infty} v^{-(s-2) q}\left(\int_{\mathbb{R}}\left(v^{-1} \int_{|\Theta| \leq v}\left|\Delta_{\Theta} f^{\prime \prime}(y)\right| \mathrm{d} \Theta\right)^{p} \mathrm{~d} y\right)^{q / p} \frac{\mathrm{d} v}{v}\right)^{1 / q} \\
& \leq c_{1} M_{p, q}^{s-2,1,1}\left(f^{\prime \prime}\right)\|g\|_{B V_{s p-1}^{s}-(1 / p)}^{1} \\
& \leq c_{2}\left\|f^{\prime}\right\|_{\mathcal{B}_{p, q}^{s-1}}\|g\|_{B V_{s p-1}^{1}}^{s-(1 / p)} .
\end{aligned}
$$

We conclude with the help of the embedding $B_{p, q}^{s} \hookrightarrow B V_{s p-1}^{1}$.

- The case $q / p<1$ and $s \geq \frac{p}{q}+\frac{1}{p}$. Proceeding as above we arrive at the inequality (4.33), i.e.,

$$
\begin{aligned}
\sum_{l} a_{l}^{p-1} & \int_{\mathbb{R}}\left(t^{-1} \int_{|\Theta| \leq a_{l} t}\left|\Delta_{\Theta} f^{\prime \prime}(y)\right| \mathrm{d} \Theta\right)^{p} \mathrm{~d} y \leq \\
& \leq\left(\sum_{l}\left\{a_{l}^{p-1} \int_{\mathbb{R}}\left(t^{-1} \int_{|\Theta| \leq a_{l} t}\left|\Delta_{\Theta} f^{\prime \prime}(y)\right| \mathrm{d} \Theta\right)^{p} \mathrm{~d} y\right\}^{q / p}\right)^{p / q}
\end{aligned}
$$

Hence,

$$
\begin{aligned}
& U_{3}^{\prime} \leq\left(\sum_{l} \int_{0}^{\infty} t^{-(s-2) q}\left\{a_{l}^{p-1} \int_{\mathbb{R}}\left(t^{-1} \int_{|\Theta| \leq a_{l} t}\left|\Delta_{\Theta} f^{\prime \prime}(y)\right| \mathrm{d} \Theta\right)^{p} \mathrm{~d} y\right\}^{q / p} \frac{\mathrm{d} t}{t}\right)^{1 / q} \\
& \leq\left(\sum_{l} a_{l}^{q(s-(1 / p))}\right)^{1 / q}\left(\int_{0}^{\infty} v^{-(s-2) q}\left(\int_{\mathbb{R}}\left(v^{-1} \int_{|\Theta| \leq v}\left|\Delta_{\Theta} f^{\prime \prime}(y)\right| \mathrm{d} \Theta\right)^{p} \mathrm{~d} y\right)^{q / p} \frac{\mathrm{d} v}{v}\right)^{1 / q} \\
& \leq c_{1} M_{p, q}^{s-2,1,1}\left(f^{\prime \prime}\right)\|g\|_{B V_{q(s-(1 / p))}^{s-(1 / p)}}^{1} \\
& \leq c_{2}\left\|f^{\prime}\right\|_{\mathcal{B}_{p, q}^{s-1}}\|g\|_{B V_{q(s-(1 / p))}^{s-(1 / p)}}^{1} .
\end{aligned}
$$

By the assumptions on $s, B_{p, q}^{s} \hookrightarrow B V_{q(s-(1 / p))}^{1}$. The desired estimate follows. 
- The case $s>1+(2 / p)$. Let us consider a number $u>1$. Using the changes of variables of (4.32), we obtain that

$$
\begin{aligned}
\int_{I_{l, t}^{\prime}}\left(t^{-1}\right. & \left.\int_{Q(x ; t)}\left|\Delta_{h}\left(f^{\prime \prime} \circ g\right)(x)\right| g^{\prime}(x+h)^{2} \mathrm{~d} h\right)^{p} \mathrm{~d} x \leq \\
& \leq c \int_{I_{l, t}^{\prime}}\left(t^{-1} \int_{Q(x ; t)}\left|\Delta_{h}\left(f^{\prime \prime} \circ g\right)(x)\right|^{u}\left|g^{\prime}(x+h)\right|^{2 u} \mathrm{~d} h\right)^{p / u} \mathrm{~d} x \\
& \leq c \int_{I_{l, t}^{\prime}}\left(t^{-1} \int_{Q(x ; t)}\left|\Delta_{h}\left(f^{\prime \prime} \circ g\right)(x)\right|^{u}\left|g^{\prime}(x)\right|^{2 u-1}\left|g^{\prime}(x+h)\right| \mathrm{d} h\right)^{p / u} \mathrm{~d} x \\
& \leq c a_{l}^{(2-(1 / u)) p-1} \int_{\mathbb{R}}\left(t^{-1} \int_{|\Theta| \leq a_{l} t}\left|\Delta_{\Theta} f^{\prime \prime}(y)\right|^{u} \mathrm{~d} \Theta\right)^{p / u} \mathrm{~d} y .
\end{aligned}
$$

Using the elementary inequality

$$
a_{l} \leq\left\|g^{\prime}\right\|_{\infty}
$$

in the integral with respect to $\Theta$, we immediately get that

$$
\begin{aligned}
U_{3}^{\prime} \leq & c_{1}\left(\sum_{l} a_{l}^{(2-(1 / u)) p-1}\right)^{1 / p}\left(\int_{0}^{\infty} t^{-(s-2) q}\right. \\
& \left.\cdot\left(\int_{\mathbb{R}}\left(t^{-1} \int_{|\Theta| \leq t\left\|g^{\prime}\right\|_{\infty}}\left|\Delta_{\Theta} f^{\prime \prime}(y)\right|^{u} \mathrm{~d} \Theta\right)^{p / u} \mathrm{~d} y\right)^{q / p} \frac{\mathrm{d} t}{t}\right)^{1 / q} \\
& \leq c_{2} M_{p, q}^{s-2,1, u}\left(f^{\prime \prime}\right)\left\|g^{\prime}\right\|_{\infty}^{s-2+(1 / u)}\|g\|_{B V_{(2-(1 / u) p-1}^{1}}^{2-(1 / u)-(1 / p)} .
\end{aligned}
$$

Assumption $s>1+(2 / p)$ allows to choose $u$ such that $1-\delta<1 / u \leq 1-(1 / p)$. Then $p \leq\left(2-\frac{1}{u}\right) p-1$. The desired estimate follows.

Estimate of $U_{3}^{\prime \prime}$. By definition of $Q(x)$, we can write

$$
U_{3}^{\prime \prime} \leq U_{7}^{\prime \prime}+2 U_{8}^{\prime \prime}
$$

where

$$
\begin{aligned}
U_{7}^{\prime \prime} & :=\left(\int_{0}^{\infty} t^{-(s-2) q}\left(\sum_{l} \int_{I_{l, t}^{\prime \prime}}\left(t^{-1} \int_{Q(x ; t)}\left|f^{\prime \prime}(g(x+h))\right| g^{\prime}(x+h)^{2} \mathrm{~d} h\right)^{p} \mathrm{~d} x\right)^{q / p} \frac{\mathrm{d} t}{t}\right)^{1 / q}, \\
U_{8}^{\prime \prime} & :=\left(\int_{0}^{\infty} t^{-(s-2) q}\left(\sum_{l} \int_{I_{l, t}^{\prime \prime}}\left|f^{\prime \prime}(g(x))\right|^{p}\left|g^{\prime}(x)\right|^{2 p} \mathrm{~d} x\right)^{q / p} \frac{\mathrm{d} t}{t}\right)^{1 / q} .
\end{aligned}
$$

Estimate of $U_{7}^{\prime \prime}$. We introduce a parameter $v>p$ whose value will be fixed later. We put

$$
\frac{1}{w}:=\frac{1}{p}-\frac{1}{v} .
$$


We define $\alpha:=(p+1) / v$, and write

$$
\begin{aligned}
\int_{I_{l, t}^{\prime \prime}}\left(t^{-1}\right. & \left.\int_{Q(x ; t)}\left|f^{\prime \prime}(g(x+h))\right| g^{\prime}(x+h)^{2} \mathrm{~d} h\right)^{p} \mathrm{~d} x \leq \\
& \leq a_{l}^{p^{2} / w} \int_{I_{l, t}^{\prime \prime}}\left|g^{\prime}(x)\right|^{p-(p / v)}\left(t^{-1} \int_{Q(x: t)}\left|f^{\prime \prime}(g(x+h))\right|\left|g^{\prime}(x+h)\right|^{\alpha} \mathrm{d} h\right)^{p} \mathrm{~d} x \\
& \leq a_{l}^{p^{2} / w} \int_{I_{l, t}^{\prime \prime}}\left|g^{\prime}(x)\right|^{p-(p / v)}\left(M\left(\left|f^{\prime \prime} \circ g\right|\left|g^{\prime}\right|^{\alpha}\right)(x)\right)^{p} \mathrm{~d} x .
\end{aligned}
$$

Applying Hölder's inequality twice and the estimate (4.13), it holds that

$$
\begin{aligned}
& \sum_{l} a_{l}^{p^{2} / w}\left(\int_{I_{l, t}^{\prime \prime}}\left|g^{\prime}(x)\right|^{(1-(1 / v)) w} \mathrm{~d} x\right)^{p / w}\left(\int_{I_{l, t}^{\prime \prime}}\left(M\left(\left|f^{\prime \prime} \circ g\right|\left|g^{\prime}\right|^{\alpha}\right)(x)\right)^{v} \mathrm{~d} x\right)^{p / v} \leq \\
& \leq\left(\sum_{l} a_{l}^{p} \int_{I_{l, t}^{\prime \prime}} \sup _{|h| \leq 2 t}\left|g^{\prime}(x+h)-g^{\prime}(x)\right|^{(1-(1 / v)) w} \mathrm{~d} x\right)^{p / w} \\
& \times\left(\sum_{l} \int_{I_{l, t}^{\prime \prime}}\left(M\left(\left|f^{\prime \prime} \circ g\right|\left|g^{\prime}\right|^{\alpha}\right)(x)\right)^{v} \mathrm{~d} x\right)^{p / v} \\
& \leq c\left(\sum_{l} a_{l}^{p}\right)^{p / w}\left(\int_{\mathbb{R}|h| \leq 2 t} \sup _{l}\left|g^{\prime}(x+h)-g^{\prime}(x)\right|^{(1-(1 / v)) w} \mathrm{~d} x\right)^{p / w} \\
& \times\left\|\left|f^{\prime \prime} \circ g\right|\left|g^{\prime}\right|^{\alpha}\right\|_{v}^{p},
\end{aligned}
$$

where the last estimate follows from the Hardy-Littlewood maximal inequality in $L_{v}$. We define

$$
s_{1}:=\frac{s-2}{1-\frac{1}{v}}, \quad p_{1}:=\left(1-\frac{1}{v}\right) w, \quad q_{1}:=\left(1-\frac{1}{v}\right) q .
$$

Applying Proposition 3.16, the estimate

$$
\left(\int_{0}^{\infty} t^{-(s-2) q}\left(\int_{\mathbb{R}} \sup _{|h| \leq 2 t}\left|g^{\prime}(x+h)-g^{\prime}(x)\right|^{p_{1}} \mathrm{~d} x\right)^{q / w} \frac{\mathrm{d} t}{t}\right)^{1 / q_{1}} \leq c\left\|g^{\prime}\right\|_{B_{p_{1}, q_{1}}^{s_{1}}}
$$

holds under the condition $1 / w<s-2<1-(1 / v)$, i.e., if and only if

$$
1-\delta<\frac{1}{v}<3-s
$$

Thus we obtain

$$
U_{7}^{\prime \prime} \leq c\left\|\left|f^{\prime \prime} \circ g\right|\left|g^{\prime}\right|^{\alpha}\right\|_{v}\|g\|_{B V_{p}^{1}}^{p / w}\left\|g^{\prime}\right\|_{B_{p_{1}, q_{1}}^{s_{1}}}^{1-(1 / v)}
$$

Since $\alpha v-1=p$, it holds that

$$
\left\|\left|f^{\prime \prime} \circ g\right|\left|g^{\prime}\right|^{\alpha}\right\|_{v} \leq\left(\sum_{l} a_{l}^{p} \int_{g\left(I_{l}\right)}\left|f^{\prime \prime}(z)\right|^{v} \mathrm{~d} z\right)^{1 / v} \leq\left\|f^{\prime \prime}\right\|_{v}\|g\|_{B V_{p}^{1}}^{p / v} .
$$


To conclude, we need the embeddings

$$
B_{p, q}^{s} \hookrightarrow B V_{p}^{1}, \quad B_{p, q}^{s-1} \hookrightarrow B_{p_{1}, q_{1}}^{s_{1}}
$$

together with the estimate

$$
\left\|f^{\prime \prime}\right\|_{v} \leq c\left\|f^{\prime}\right\|_{\mathcal{B}_{p, q}^{s-1}} .
$$

The first embedding in (4.38) follows from $s>1+(1 / p)$, and the second from

$$
\delta \geq \frac{1}{1-\frac{1}{v}}\left(\delta-1+\frac{1}{v}\right) .
$$

According to Proposition 3.23, inequality (4.39) follows from

$$
1-\delta<\frac{1}{v}<\frac{1}{(s-1) p} .
$$

Combining (4.37), (4.40) and (4.41), we see that the number $v$ must satisfy

$$
1-\delta<\frac{1}{v}<\min \left(\frac{1}{p}, \frac{1}{1+\delta}, \frac{1}{(s-1) p}, 3-s\right) .
$$

The existence of such a number follows from the conditions $s>2, p>1$ and $0<\delta<1$. Since $(4.37)$ implies $2-(1 / v)<s-(1 / p)$, it holds that

$$
U_{7}^{\prime \prime} \leq c_{1}\left\|f^{\prime}\right\|_{\mathcal{B}_{p, q}^{s-1}}\left\|g^{\prime}\right\|_{B_{p, q}^{s-1}}^{2-(1 / v)} \leq c_{2} V(f ; g) .
$$

Estimate of $U_{8}^{\prime \prime}$. Using Hölder's inequality (with $v$ and $w$, see (4.35)) and (4.13), we obtain that

$$
\begin{aligned}
& \int_{I_{l, t}^{\prime \prime}}\left|f^{\prime \prime}(g(x))\right|^{p}\left|g^{\prime}(x)\right|^{2 p} \mathrm{~d} x \leq \\
& \leq a_{l}^{p} \int_{I_{l, t}^{\prime \prime}}\left|f^{\prime \prime}(g(x))\right|^{p}\left|g^{\prime}(x)\right|^{p / v} \sup _{|h| \leq 2 t}\left|g^{\prime}(x+h)-g^{\prime}(x)\right|^{p-(p / v)} \mathrm{d} x \\
& \leq a_{l}^{p}\left(\int_{g\left(I_{l, t}^{\prime \prime}\right)}\left|f^{\prime \prime}(z)\right|^{v} \mathrm{~d} z\right)^{p / v} \cdot\left(\int_{I_{l, t}^{\prime \prime}|h| \leq 2 t} \sup \left|g^{\prime}(x+h)-g^{\prime}(x)\right|^{(1-(1 / v)) w} \mathrm{~d} x\right)^{p / w} \\
& \leq a_{l}^{p}\left\|f^{\prime \prime}\right\|_{v}^{p}\left(\int_{\mathbb{R}} \sup _{|h| \leq 2 t}\left|g^{\prime}(x+h)-g^{\prime}(x)\right|^{(1-(1 / v)) w} \mathrm{~d} x\right)^{p / w} .
\end{aligned}
$$

So

$$
U_{8}^{\prime \prime} \leq c\left\|f^{\prime \prime}\right\|_{v}\|g\|_{B V_{p}^{1}}\left\|g^{\prime}\right\|_{B_{p_{1}, q_{1}}^{s_{1}}}^{1-(1 / v)}
$$

where $s_{1}, p_{1}$ and $q_{1}$ are defined in (4.36). Now the estimate of $U_{8}^{\prime \prime}$ can be obtained as that of $U_{7}^{\prime \prime}$. We omit the details.

Substep 2.2: Estimate of $U_{4}$. We write $U_{4} \leq U_{4}^{\prime}+U_{4}^{\prime \prime}$, as in (4.30), where $U_{4}^{\prime}$ and $U_{4}^{\prime \prime}$ are the corresponding terms with respect to the decomposition using the 
families $\left\{I_{l, t}^{\prime}\right\}_{l}$ and $\left\{I_{l, t}^{\prime \prime}\right\}_{l}$, respectively.

- In the estimate of $U_{4}^{\prime}$, we just replace (4.31) by

$$
g^{\prime}(x)^{2} \leq\left|g^{\prime}(x)\right|\left|g^{\prime}(x+h)\right|, \quad \forall h \in P(x) .
$$

- As in (4.34), we decompose $U_{4}^{\prime \prime} \leq U_{9}^{\prime \prime}+2 U_{10}^{\prime \prime}$ where

$$
\begin{aligned}
U_{9}^{\prime \prime} & :=\left(\int_{0}^{\infty} t^{-(s-2) q}\left(\sum_{l} \int_{I_{l, t}^{\prime \prime}}\left(t^{-1} \int_{P(x ; t)}\left|f^{\prime \prime}(g(x+h))\right| g^{\prime}(x)^{2} \mathrm{~d} h\right)^{p} \mathrm{~d} x\right)^{q / p} \frac{\mathrm{d} t}{t}\right)^{1 / q}, \\
U_{10}^{\prime \prime} & :=\left(\int_{0}^{\infty} t^{-(s-2) q}\left(\sum_{l} \int_{I_{l, t}^{\prime \prime}}\left|f^{\prime \prime}(g(x))\right|^{p}\left|g^{\prime}(x)\right|^{2 p} \mathrm{~d} x\right)^{q / p} \frac{\mathrm{d} t}{t}\right)^{1 / q} .
\end{aligned}
$$

Then the estimate of $U_{9}^{\prime \prime}$ (resp. $U_{10}^{\prime \prime}$ ) is similar to that of $U_{7}^{\prime \prime}$ (resp. $U_{8}^{\prime \prime}$ ).

Substep 2.3: Estimate of $U_{1}$. Let $0<\alpha<1$. Using notation (4.35) and Hölder's inequality with $(1-\alpha)+\alpha=1$, we obtain

$$
\begin{aligned}
& \int_{\mathbb{R}}\left(t^{-1} \int_{Q(x ; t)}\left|f^{\prime \prime}(g(x))\right|\left|\Delta_{h}\left(g^{\prime 2}\right)(x)\right| \mathrm{d} h\right)^{p} \mathrm{~d} x \leq \\
& \quad \leq c_{1} \int_{\mathbb{R}}\left(t^{-1} \int_{Q(x ; t)}\left|g^{\prime}(x+h)-g^{\prime}(x)\right|^{1-\alpha} \mathrm{d} h\right)^{p}\left|f^{\prime \prime}(g(x))\right|^{p}\left|g^{\prime}(x)\right|^{p(1+\alpha)} \mathrm{d} x \\
& \quad \leq c_{2}\left(\int_{\mathbb{R}}\left(t^{-1} \int_{-t}^{t}\left|g^{\prime}(x+h)-g^{\prime}(x)\right| \mathrm{d} h\right)^{w(1-\alpha)} \mathrm{d} x\right)^{p / w}\left\|\left(f^{\prime \prime} \circ g\right)\left|g^{\prime}\right|^{1+\alpha}\right\|_{v}^{p} .
\end{aligned}
$$

Arguing as in the estimate of $U_{7}^{\prime \prime}$, we deduce that

$$
U_{1} \leq c\left\|f^{\prime \prime}\right\|_{v}\|g\|_{B V_{(1+\alpha) v-1}^{1+\alpha-(1 / v)}}^{1+\alpha} g^{\prime} \|_{B_{p_{1}, q_{1}}^{s_{1}}}^{1-\alpha}
$$

where

$$
s_{1}=\frac{s-2}{1-\alpha}, \quad p_{1}=(1-\alpha) w, \quad q_{1}=(1-\alpha) q,
$$

under condition $0<s_{1}<1$ and $p_{1} \geq 1$. The first condition is equivalent to

$$
\alpha<3-s,
$$

the second will be satisfied by the future choice of $v$. Now we need the estimate (4.39) for $\left\|f^{\prime \prime}\right\|_{v}$; this follows from condition (4.41). We need also the embeddings $B_{p, q}^{s-1} \hookrightarrow B_{p_{1}, q_{1}}^{s_{1}}$ and $B_{p, q}^{s} \hookrightarrow B V_{(1+\alpha) v-1}^{1}$, which come from

$$
\delta \geq s_{1}-\frac{1}{p_{1}}, \quad p_{1} \geq p, \quad p \leq(1+\alpha) v-1 .
$$

Combining (4.41) and (4.45), we obtain the following condition for $1 / v$ :

$$
\max \left(1-\delta, \frac{\alpha}{p}\right)<\frac{1}{v}<\min \left(1-\alpha \delta, \frac{1+\alpha}{1+p}, \frac{1}{(s-1) p}\right) .
$$


Notice that $(3-s)(s-1)<1$ for $s \neq 2$. Hence (4.44) implies $\alpha<\frac{1}{s-1}$ and

$$
\frac{\alpha}{p}<\min \left(1-\alpha \delta, \frac{1}{(s-1) p}\right),
$$

while $\frac{\alpha}{p}<\frac{1+\alpha}{1+p}$ follows by $\alpha<1<p$. Inequality $1-\delta<1-\alpha \delta$ follows from $\alpha<1$, while $1-\delta<\frac{1}{(s-1) p}$ was observed in the estimate of $U_{7}^{\prime \prime}$. Inequality $1-\delta<\frac{1+\alpha}{1+p}$ is equivalent to

$$
p-(p+1) \delta<\alpha
$$

The condition $s>2$ guarantees the compatibility between (4.44) and (4.47). Thus it is possible to find $v$ satisfying (4.46). For such a $v$, we obtain that

$$
U_{1} \leq c_{1}\left\|f^{\prime}\right\|_{\mathcal{B}_{p, q}^{s-1}}\|g\|_{B_{p, q}^{s}}^{2-(1 / v)} \leq c_{2} V(f ; g) .
$$

The last inequality follows from $1-\delta<1 / v$.

Substep 2.4: Estimate of $U_{2}$. Let $0<\alpha<1$ and $1<v_{1}<v$ (see (4.35) for the definition of $v$ ). We put

$$
\frac{1}{w_{1}}:=1-\frac{1}{v_{1}}
$$

Using the following elementary inequality:

$$
\left|\Delta_{h}\left(g^{\prime 2}\right)(x)\right| \leq c\left|g^{\prime}(x+h)\right|^{1+\alpha}\left|\Delta_{h} g^{\prime}(x)\right|^{1-\alpha}, \quad \forall h \in P(x),
$$

and by Hölder inequality applied three times (in particular with $(1-\alpha)+\alpha=1$ ), we obtain that

$$
\begin{aligned}
& \int_{\mathbb{R}}\left(t^{-1} \int_{P(x ; t)}\left|f^{\prime \prime}(g(x+h))\right|\left|\Delta_{h}\left(g^{\prime 2}\right)(x)\right| \mathrm{d} h\right)^{p} \mathrm{~d} x \leq \\
& \leq c_{1} \int_{\mathbb{R}}\left(M\left(\left.\left.\left|\left(f^{\prime \prime} \circ g\right)\right| g^{\prime}\right|^{1+\alpha}\right|^{v_{1}}\right)(x)\right)^{p / v_{1}}\left(t^{-1} \int_{-t}^{t}\left|\Delta_{h} g^{\prime}(x)\right|^{w_{1}(1-\alpha)} \mathrm{d} h\right)^{p / w_{1}} \mathrm{~d} x \\
& \leq c_{2}\left\|M\left(\left.\left.\left|\left(f^{\prime \prime} \circ g\right)\right| g^{\prime}\right|^{1+\alpha}\right|^{v_{1}}\right)\right\|_{v / v_{1}}^{p / v_{1}}\left(\int_{\mathbb{R}}\left(t^{-1} \int_{-t}^{t}\left|\Delta_{h} g^{\prime}(x)\right|^{w_{1}} \mathrm{~d} h\right)^{(1-\alpha) w / w_{1}} \mathrm{~d} x\right)^{p / w} .
\end{aligned}
$$

Using the notation (4.43) for $s_{1}, p_{1}$ and $q_{1}$, it holds that

$$
U_{2} \leq c\left\|\left(f^{\prime \prime} \circ g\right)\left|g^{\prime}\right|^{1+\alpha}\right\|_{v}\left\|g^{\prime}\right\|_{B_{p_{1}, q_{1}}^{s_{1}}}^{1-\alpha} .
$$

Now we choose a number $v$ as in (4.46). An analogous estimate to the one obtained for $U_{1}$ (see (4.42) and (4.48)) allows us to get that $U_{2} \leq c V(f ; g)$. Notice that the condition

$$
\frac{1}{p_{1}}-\frac{1}{w_{1}}<s_{1}<1
$$

is guaranteed by (4.44) and $1-\delta<1 / v$. 


\section{Proof of Theorem 2.1 and Corollary 2.3}

\subsection{Proof of Corollary 2.3}

Using Propositions 3.15, 3.10, 3.21(ii), Theorem 2.2 and condition $f(0)=0$, we derive

$$
\begin{aligned}
\|f \circ g\|_{B_{p, q}^{s}} & \leq c_{1}\left(\|f \circ g\|_{p}+\left\|(f \circ g)^{\prime}\right\|_{\dot{B}_{p, q}^{s-1}}\right) \\
& \leq c_{2}\left(\left\|f^{\prime}\right\|_{\infty}\|g\|_{p}+\left\|f^{\prime}\right\|_{\mathcal{B}_{p, q}^{s-1}}\left(\|g\|_{B_{p, q}^{s}}+\|g\|_{B_{p, q}^{s}}^{s-(1 / p)}\right)\right) \\
& \leq c_{3}\left\|f^{\prime}\right\|_{\mathcal{B}_{p, q}^{s-1}}\left(\|g\|_{B_{p, q}^{s}}+\|g\|_{B_{p, q}^{s}}^{s-(1 / p)}\right) .
\end{aligned}
$$

This proves (i).

We now prove (ii). It holds that $f \circ g=\left(f \rho_{t}\right) \circ g$ for $t:=\|g\|_{\infty}$. By the embedding $B_{p, q}^{s-1} \hookrightarrow \mathcal{B}_{p, q}^{s-1}$, see Proposition 3.21(i), and by the assumption on $f$, it holds that

$$
\left\|\left(f \rho_{t}\right)^{\prime}\right\|_{\mathcal{B}_{p, q}^{s-1}} \leq c\left\|\left(f \rho_{t}\right)^{\prime}\right\|_{B_{p, q}^{s-1}} \leq c\left\|f \rho_{t}\right\|_{B_{p, q}^{s}}<+\infty .
$$

Since $\left(f \rho_{t}\right)(0)=0$, the desired inequality follows from (i).

\subsection{Proof of Theorem 2.1}

Proof of (i). As mentioned in the Introduction, the necessity of $f(0)=0$ and $f \in B_{p, q}^{s, \ell o c}$ is well known. The sufficiency part of Theorem 2.1 is an immediate consequence of Corollary 2.3 (ii).

Proof of (ii). Boundedness follows immediately from the embedding $B_{p, q}^{s} \hookrightarrow C_{b}$, see Proposition 3.21(i) and Corollary 2.3(ii). The fact that $T_{f}$ is not sublinear, except in case $f$ is linear, has been proved in Proposition 11 of [4]; this statement follows also from Proposition 6.1 (see below) and a homogeneity argument.

\section{Optimality of the estimates}

From the condition $s-(1 / p)>1$, the inequality (2.2) implies that

$$
\|f \circ g\|_{B_{p, q}^{s}} \leq c\left\|f^{\prime}\right\|_{\mathcal{B}_{p, q}^{s-1}}\left(1+\|g\|_{B_{p, q}^{s}}\right)^{s-(1 / p)}
$$

Now the exponent $s-(1 / p)$ in $(6.1)$ is optimal except if $f$ is a polynomial. Indeed we have the following statement:

Proposition 6.1. Let $0<\alpha<s-(1 / p)$ and let $N$ be any seminorm on $\mathcal{D}$. If $f$ is a continuous function such that, for some constant $c=c(f)>0$, the inequality

$$
\|f \circ g\|_{B_{p, q}^{s}} \leq c(1+N(g))^{\alpha}
$$

holds for all $g \in \mathcal{D}$, then $f$ is a polynomial of degree $\leq s$. 
Proof. We use the same argument as in the proofs of Propositions 11 and 12 in [4]. Let us consider the integer $m \geq 1$ such that $m-1 \leq s<m$. Let us define $\varphi \in \mathcal{D}$ by $\varphi(x):=x \rho(x), \forall x \in \mathbb{R}$, see (1.1) for the definition of $\rho$. Then it holds that $(f \circ a \varphi)(x)=f(a x)$ for all $x \in[-1,1]$ and all $a>0$. Hence,

$$
\Delta_{t}^{m}(f \circ a \varphi)(x)=\Delta_{a t}^{m} f(a x), \quad \forall x \in[-1 / 2,1 / 2], \forall t \in[0,1 / 2 m], \forall a>0 .
$$

From the embedding $B_{p, q}^{s} \hookrightarrow B_{p, \infty}^{s},(6.2)$ and (6.3), we deduce that

$$
\left.\left.\left(\int_{-a / 2}^{a / 2}\left|\Delta_{t}^{m} f(x)\right|^{p} \mathrm{~d} x\right)^{1 / p} \leq c a^{\alpha+(1 / p)-s} t^{s}, \quad \forall t \in\right] 0,1\right], \forall a>2 m .
$$

Applying the assumption on $\alpha$, and taking $a \rightarrow+\infty$, we obtain that

$$
\left.\left.\Delta_{t}^{m} f(x)=0 \quad \text { a.e. }, \quad \forall t \in\right] 0,1\right]
$$

Then, using a standard argument, we deduce that $f$ is a polynomial of degree at most $m-1$.

Acknowledgements. I would like to thank Gérard Bourdaud and Winfried Sickel for their support and encouragement to write this article. Thanks are also due to the referee for his remarks which led to an improvement of the paper.

\section{References}

[1] Allaoui, S. E.: Remarques sur le calcul symbolique dans certains espaces de Besov à valeurs vectorielles. Ann. Math. Blaise Pascal 16 (2009), 399-429.

[2] Bourdaud, G.: Le calcul fonctionnel dans les espaces de Sobolev. Invent. Math. 104 (1991), 435-446.

[3] Bourdaud, G.: Fonctions qui opèrent sur les espaces de Besov et de Triebel. Ann. Inst. H. Poincaré Anal. Non Linéaire 10 (1993), no. 4, 413-422.

[4] Bourdaud, G., Lanza de Cristoforis, M. and Sickel, W.: Superposition operators and functions of bounded p-variation. Rev. Mat. Iberoam. 22 (2006), 455-487.

[5] Bourdaud, G. And Meyer, Y.: Le calcul fonctionnel sous-linéaire dans les espaces de Besov homogènes. Rev. Mat. Iberoam. 22 (2006), 725-746.

[6] Bourdaud, G., Moussai, M. And Sickel, W.: An optimal symbolic calculus on Besov algebras. Ann. Inst. H. Poincaré Anal. Non Lineáire 23 (2006), 949-956.

[7] Bourdaud, G., Moussai, M. and Sickel, W.: Towards sharp superposition theorems in Besov and Lizorkin-Triebel spaces. Nonlinear Anal. 68 (2008), 2889-2912.

[8] Bourdaud, G., Moussai, M. And Sickel, W.: Composition operators on Lizorkin-Triebel spaces. J. Funct. Anal. 259 (2010), 1098-1128.

[9] DahlberG, B. E. J.: A note on Sobolev spaces. In Harmonic analysis in Euclidean spaces (Proc. Sympos. Pure Math., Williams Coll., Williamstown, Mass., 1978), Part 1, 183-185. Proc. Symp. Pure Math. XXXV, Amer. Math. Soc., Providence, RI, 1979. 
[10] Franke, J.: On the spaces $\mathbf{F}_{p q}^{s}$ of Triebel-Lizorkin type: Pointwise multipliers and spaces on domains. Math. Nachr. 125 (1986), 29-68.

[11] Marcus, M. And Mizel, V. J.: Every superposition operator mapping one Sobolev space into another is continuous. J. Funct. Anal. 33 (1979), 217-229.

[12] Meyer, Y.: Régularité des solutions des équations aux derivées partielles non linéaires (d'après J. M. Bony). In Bourbaki Seminar, Vol. 1979/80, 293-302. Lecture Notes in Math. 842, Springer, Berlin-New York, 1981.

[13] Peetre, J.: Interpolation of Lipschitz operators and metric spaces. Mathematica (Cluj) 12 (1970), 325-334.

[14] Peetre, J.: New thoughts on Besov spaces. Duke University Mathematics Series I, Mathematics Department, Duke University, Durham, NC, 1976.

[15] Runst, T.: Mapping properties of nonlinear operators in spaces of Triebel-Lizorkin and Besov type. Anal. Math. 12 (1986), 313-346.

[16] Runst, T. And Sickel, W.: Sobolev spaces of fractional order, Nemytskij operators and nonlinear partial differential equations. De Gruyter Series in Nonlinear Analysis and Applications 3, Walter de Gruyter, Berlin, 1996.

[17] TrieBel, H.: Theory of function spaces. Monographs in Mathematics 78, Birkhäuser Verlag, Basel, 1983.

[18] Triebel, H.: Theory of function spaces. II. Monographs in Mathematics 84, Birkhäuser Verlag, Basel, 1992.

[19] Yamazaki, M.: A quasihomogeneous version of paradifferential operators. I: Boundedness on spaces of Besov type. J. Fac. Sci. Univ. Tokyo Sect. IA Math. 33 (1986), 131-174. II: A symbolic calculus. J. Fac. Sci. Univ. Tokyo Sect. IA Math. 33 (1986), 311-345.

[20] Wiener, N.: The quadratic variation of a function and its Fourier coefficients. J. Math. Phys. 3 (1924), 72-94.

Received April 11, 2010; revised February 13, 2011.

Madani Moussai: Department of Mathematics, Lab. Math. Pure and Applied, University of M'Sila, P. O. Box 166, M'Sila 28000, Algeria.

E-mail: mmoussai@yahoo.fr 